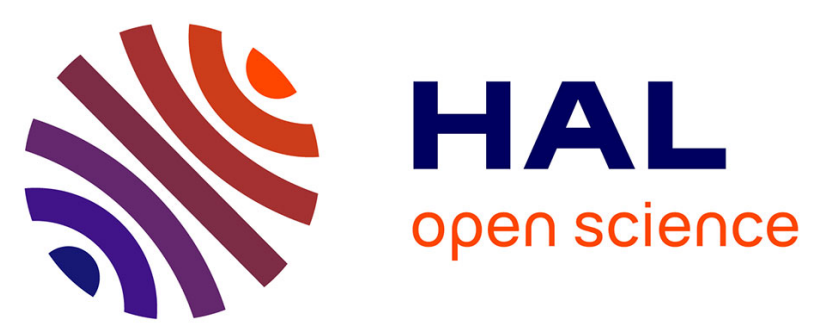

\title{
MARMIT: A multilayer radiative transfer model of soil reflectance to estimate surface soil moisture content in the solar domain (400-2500 $\mathrm{nm})$
}

\author{
Aurelien Bablet, P.V.H. V H Vu, Stéphane Jacquemoud, Françoise
}

Viallefont-Robinet, Sophie Fabre, Xavier Briottet, Morteza Sadeghi, Michael

L. Whiting, Frédéric Baret, Jia Tian

\section{To cite this version:}

Aurelien Bablet, P.V.H. V H Vu, Stéphane Jacquemoud, Françoise Viallefont-Robinet, Sophie Fabre, et al.. MARMIT: A multilayer radiative transfer model of soil reflectance to estimate surface soil moisture content in the solar domain $(400-2500 \mathrm{~nm})$. Remote Sensing of Environment, 2018, 217, pp.1-17. 10.1016/j.rse.2018.07.031 . hal-02118848

\section{HAL Id: hal-02118848 \\ https://hal.science/hal-02118848}

Submitted on 3 May 2019

HAL is a multi-disciplinary open access archive for the deposit and dissemination of scientific research documents, whether they are published or not. The documents may come from teaching and research institutions in France or abroad, or from public or private research centers.
L'archive ouverte pluridisciplinaire HAL, est destinée au dépôt et à la diffusion de documents scientifiques de niveau recherche, publiés ou non, émanant des établissements d'enseignement et de recherche français ou étrangers, des laboratoires publics ou privés. 
Date: Monday, June 4, 2018

Title: MARMIT: a multilayer radiative transfer model of soil reflectance to estimate surface soil moisture content in the solar domain $(400-2500 \mathrm{~nm})$

Authors: A. Bablet ${ }^{1,2, *}$, P.V.H. Vu ${ }^{2,3}$, S. Jacquemoud ${ }^{2}$, F. Viallefont-Robinet ${ }^{1}$, S. Fabre ${ }^{1}$, X. Briottet ${ }^{1}$, M. Sadeghi ${ }^{4}$, M.L. Whiting ${ }^{5}$, F. Baret ${ }^{6}$ and J. Tian ${ }^{7}$

1 ONERA/DOTA, Université de Toulouse, 2 avenue Edouard Belin, BP 74025, 31055 Toulouse Cedex 4, France (aurelien.bablet@onera.fr, francoise.viallefont@onera.fr, sophie.fabre@onera.fr, xavier.briottet@onera.fr)

2 Institut de physique du globe de Paris - Sorbonne Paris Cité, Université Paris Diderot, UMR CNRS 7154, Case 7071, 35-39 rue Hélène Brion, 75013 Paris, France (jacquemoud@ipgp.fr)

3 University of Science and Technology of Hanoi, 18 Hoàng Quốc Việt, Nghĩa Đô, Cầu Giấy, Hà Nội, Vietnam (vuphan.viethoa@gmail.com)

4 Department of Plants, Soils and Climate, Utah State University, 4820 Old Main Hill, Logan, UT 84322-4820, USA (morteza.sadeghi@usu.edu)

5 CSTARS, Department of Land, Air, and Water Resources, University of California, One Shield Avenue, Davis, CA 95616, USA (mwhiting@ucdavis.edu)

6 UMR EMMAH, INRA, UAPV, 228 route de l'Aérodrome, 84914 Avignon Cedex 9, France (frederic.baret@inra.fr)

7 Department of Civil and Environmental Engineering, Cornell University, Ithaca, NY 14853, USA (jt636@cornell.edu)

${ }^{*}$ Corresponding author

Keywords: Remote sensing, Soil moisture content, Reflectance spectroscopy, Radiative transfer model, Spectral signature

\section{Highlights:}

- A multilayer radiative transfer model of soil reflectance as a function of surface water content is developed

- A new method of SMC retrieval is developed

- SMC retrieval combines good accuracy and efficiency after a soil classification

- The new method is compared to other SMC retrieval methods

Journal: Remote Sensing of Environment

\section{Abstract}

Surface soil moisture content (SMC) is known to impact soil reflectance at all wavelengths of the solar spectrum. As a consequence, many semi-empirical methods aim at inferring SMC from soil reflectance, but very few rely on physically-based models. This article presents a multilayer radiative transfer model of soil reflectance called MARMIT (multilayer radiative transfer model of soil reflectance) as a function of SMC given on a mass basis and a method called MARMITforSMC to estimate it from soil reflectance spectra. This model depicts a wet soil as a dry soil covered with a thin film of water. It is used to assess SMC over seven independent laboratory datasets gathered from the literature. A learning phase is required to link the thickness of the water film with the SMC. For that purpose, a sigmoid function, the parameters of which are related to soil physical and chemical properties such as porosity, grain size and mineralogy composition, is fitted. SMC can be inferred with good accuracy (RMSE $\approx 3 \%$ ) if the learning step is applied soil by soil. The link between SMC 
and water thickness actually depends on soil texture and chemical composition. If the soils are divided into classes and if the learning phase is applied to a class, the RMSE slightly increases up to 5\%. Finally, MARMITforSMC provides lower RMSE than any other existing semi-empirical or physically-based method.

\section{Introduction}

Soil water content (or soil moisture content, SMC) assessment is critical in agriculture, hydrology, micrometeorology, defense, civil engineering, and other environmental fields (e.g., Gardner, 2000; Robinson et al., 2008; Vereecken et al., 2008; Wang and Qu, 2009; Ochsner et al., 2013). In agriculture, SMC is an indicator of soil sensitivity to wind erosion; it also provides information about water infiltration, runoff and storage that helps monitor soil-water-plant conditions and manage irrigation (Glenn et al., 2009; Yang et al., 2012). Therefore, it is highly correlated with crop yield estimation. In hydrology and meteorology, SMC plays an important role in flood prevention (Haubrock et al., 2008), incident radiation distribution and, indirectly, temperature and evaporation (Khanna et al., 2007; Patel et al., 2009); thus it contributes to mass conservation and energy balance calculation. In defense or homeland security, trafficability depends on surface characteristics including SMC and can be key in succeeding military or humanitarian operations. Vehicle traffic is easier on dry clay paths and, conversely, on wet sand paths. SMC is also increasingly examined in planetary sciences: for instance, the reflectance of some Martian surfaces in the near-infrared presents strong absorption features attributed to the presence of water in the regolith or in the minerals that compose it (e.g., Milliken and Mustard, 2005, 2007a,b; Pommerol et al., 2009, 2013). Last but not least, water alters the background reflectance of a bare soil and the apparent mineral absorption depths in the spectrum, therefore it affects classification accuracy. Determining soil moisture content from reflectance measurements may be useful to quantify other information of interest such as mineralogy, salinity, texture, organic matter content or roughness (e.g., Ben Dor et al., 2002; Whiting, 2004; Bogrekci and Lee, 2006; Minasny et al., 2011; Rienzi et al., 2014; Rodionov et al., 2014; Zu et al., 2016; Marion and Carrère, 2018). 
There are three main types of soil water: (1) hydration (absorbed) water incorporated into the lattice of minerals; (2) hygroscopic (adsorbed) water bound to soil particles, including soil organic matter, due to the attraction between surface electrical charges and water molecules; (3) free water covering the minerals, occupying the pores and moving through the soil by gravity and capillary forces.

Soil water content generally refers to mass or volumetric water content, both expressed as a fraction. As stated by Stafford (1988) or Petropoulos et al. (2013), there is no conventional method to determine SMC. It is measured either in the laboratory with gravimetric and thermogravimetric methods or in the field using, for example, portable neutron probes, time domain reflectometry (TDR) or capacitance probes. Such measurements are reliable, but their footprint is limited to a few square meters at most. Moreover, soil water content abruptly varies both in space and time due to the spatial variability of soil physical properties and to the discontinuous nature of rainfall. All these methods may be expensive and laborious to implement, especially at a fine spatial sampling interval, when a large number of measurements are required.

Remote sensing can provide data at different spatial resolutions at reasonable costs. Moreover, it is a non-destructive and non-invasive method. Most researches have focused on the measurement of the backscattering coefficient and the brightness temperature in the microwave domain (Njoku and Entekhabi, 1996; Das et al., 2008; Mladenova et al., 2014) that allow determining the volumetric water content in the first centimeters, especially at lower frequencies (Tabatabaeenejad et al., 2015). But it often requires extra information about the soil dielectric constant or the surface roughness. In the solar domain (400-2500 nm), light penetration in soil varies from a few micrometers to a few millimeters depending on the wavelength and the soil type. Remote sensing offers the possibility to determine moisture of the topmost layer of the soil with much higher spatial resolution (Sadeghi et al., 2017) and may be an indicator of moisture below in some unique profiles.

Water spectroscopy is well known but still complex: the main absorption features of liquid water occurring in the infrared result from vibrational transitions involving various overtones and combinations of three fundamental vibrational transitions at $2870 \mathrm{~nm}$ (asymmetric $\mathrm{O}-\mathrm{H}$ stretching), 
$3050 \mathrm{~nm}$ (symmetric $\mathrm{O}-\mathrm{H}$ stretching), and $6080 \mathrm{~nm}(\mathrm{O}-\mathrm{H}$ bending). In the shortwave infrared, two

102

103

104

105

106

107

108

109

110

111

112

113

114

115

major water absorption peaks centered at $1470 \mathrm{~nm}$ and $1900 \mathrm{~nm}$, and two minor absorption peaks centered at $970 \mathrm{~nm}$ and $1200 \mathrm{~nm}$, are observed. Smaller peaks and shoulders can be found in the visible-near infrared at 514 nm, 606 nm, 660 nm, 739 nm, and 836 nm (Eisenberg and Kauzmann, 2005; Wozniak and Dera, 2007). The dominant effect of water on soil optical properties is an overall decrease in spectral reflectance with increasing soil moisture. Idso et al. (1975) provided empirical evidence that soil albedo decreased linearly with soil moisture, but subsequent studies have challenged this view (Bowers and Hanks, 1965; Bedidi et al., 1992; Muller and Décamps, 2001). Indeed, as is common in optics, nonlinear phenomena induce more pronounced absorption features in the absorption bands of water than any others. For high SMC, soil reflectance may even increase with moisture due to specular reflection (Neema et al., 1987; Liu et al., 2002). That critical point, which seems to correspond to the field capacity, strongly depends on soil type (Liu et al., 2002). Soil reflectance is controlled by many other factors such as texture, mineralogy, organic matter, and surface roughness (e.g, Clark and Roush, 1984; Baumgardner et al., 1985; Okin and Painter, 2004; Stenberg et al., 2010; Ben-Dor, 2011).

Numerous experiments have measured soil reflectance variation as a function of soil moisture in the visible (VIS, 0.4-0.7 $\mu \mathrm{m}$ ), in the near-infrared (NIR, 0.7-1.0 $\mu \mathrm{m}$ ), in the shortwave-infrared (SWIR, 1.0-3.0 m) (e.g., Bowers and Hanks, 1965; Skidmore et al., 1968; Planet, 1970; Idso et al., 1975; Twomey et al., 1986; Ishida et al., 1991; Liu et al., 2002; Haubrock et al., 2008), and more recently, in the midwave-infrared (MWIR, 3.0-8.0 $\mu \mathrm{m}$ ) and longwave-infrared (LWIR, 8.0-12.0 $\mu \mathrm{m}$ ) (e.g., Van Bavel et al., 1976; Narayanan et al., 1993; Bishop et al., 1994; Mira et al., 2007; Lesaignoux et al., 2013). Many empirical methods link SMC and reflectance. They include spectral indices (e.g., Levitt et al., 1990; Bryant et al., 2003; Khanna et al., 2007; Haubrock et al., 2008; Gao et al., 2013), statistical relationships (e.g., Lesaignoux et al., 2013; Yin et al., 2013), multivariate analysis (Mouazen et al., 2006), wavelet analysis (Peng et al., 2013) and exponential functions (Muller and Décamps, 2001; Liu et al., 2002; Lobell and Asner, 2002; Whiting et al., 2004; Kaleita et al., 2005; Sun et al., 2007; Somers et al., 2010; Verpoorter et al., 2014; Zhang et al., 2014). Such 
methods have been applied to reflectance spectra and their successive derivatives, and to continuumremoved spectra. However, most of them are not universal because they have been calibrated over a limited range of soil types and knowledge of parameters such as soil density or porosity may be required.

Very few methods relying on physically-based models have been developed. In the 1920s, Ångström (1925) proposed a simple model where a wet soil is regarded as a dry soil covered with a thin film of liquid water. This model derives the albedo of a wet soil by calculating the multiple reflections between the two media based on Snell's law. Lekner and Dorf (1988) improved the Ångström model by using the Fresnel coefficients instead of Snell’s law. Bach and Mauser (1994) introduced the Beer-Lambert-Bouguer law to account for light absorption in the water layer and extended the model to the VIS-SWIR. More recently, Kimmel and Baranoski (2007) published a ray tracing model called SPLITS (spectral light transport model for sand) and Sadeghi et al (2015) proposed a model based on the Kubelka-Munk two-flux radiative transfer model. However, SPLITS requires information on the soil that is somewhat difficult to access, high computing resources, and it does not allow retrieving the SMC. As for the Sadeghi model, it only works at some wavelengths, which reduces its field of application (see Section 4.3).

In this article, we improve the Bach model (Bach and Mauser, 1994) which appears to efficiently estimate the surface SMC $\left(r^{2}=0.88\right)$ but which has not been validated or improved in the literature for the past twenty years. The equations underlying the multilayer radiative transfer model of soil reflectance (MARMIT) are detailed, and the validation datasets are presented. Then, a method to retrieve SMC called MARMITforSMC and based on a logistic function is introduced and compared to other statistical or semi-empirical methods.

\section{Model and datasets}

\subsection{Description of MARMIT}



approach is naturally a simplified version of reality, as the geometry of water films within soils is much more complex (Tuller et al., 1999). A fraction of light is transmitted from the air (medium 1) to reflectivity at the interface is $r_{12}=1-t_{12}$. Then light is diffusely scattered through internal multiple reflections between the liquid-soil interface $\left(R_{d}\right)$ and the liquid-air interface $\left(r_{21}\right)$. These multiple reflections increase the probability of light absorption by soil particles and explain why a wet soil from the water to the air $\left(t_{12}\right)$.

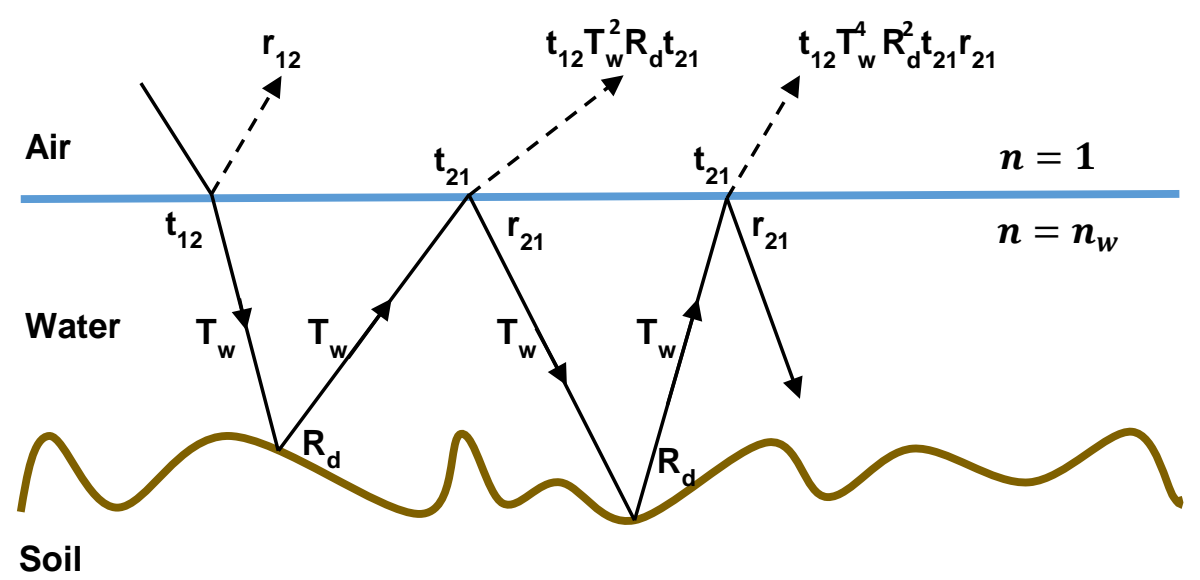

Fig. 1. Thin liquid water layer over a rough surface. Medium 1 is air and medium 2 is water. $r_{i j}$ and $t_{i j}$ are the reflectivity and transmissivity at the interface between the media $i$ and $j, T_{w}$ is the transmittance of the water layer, $R_{d}$ the reflectance of the dry soil. $n_{w}$ is the refractive index of water, that of air being assumed to be 1 . All these physical quantities are wavelength dependent.

MARMIT results from a series of improvements of an approach initiated by Ångström (1925),

$$
A_{w s L}=\frac{t_{12} A_{d}}{1-r_{21} R_{d}}
$$


171 where $A_{d}=1-R_{d}$ is the absorptance of the dry soil, $t_{12}$ the transmissivity at the air-water interface 172 and $r_{21}$ the reflection coefficient at the water-air interface. Both are calculated by the Fresnel 173 equations for unpolarized light. $t_{12}$ depends on the incidence angle $\theta_{i}$ formed between the normal and 174 the incident ray and on the relative refractive index $n$ defined as the ratio of the refractive index of 175 pure liquid water $\left(n_{w}\right)$ to the refractive index of the air $\left(n_{a}=1\right): n=n_{w} / n_{a}=n_{w}$. Ångström 176 (1925) assumes that light exiting the water layer lies within a cone of a given angle while Lekner and 177 Dorf (1988) consider a diffuse light. The latter consequently calculate $r_{21}$ by integrating the 178 reflectivity over the entire hemisphere (Stern, 1964):

$$
r_{21}=1-\frac{1}{n^{2}}\left(1-r_{12}^{\prime}\right)
$$

181 with

$$
r_{12}^{\prime}=\frac{3 n^{2}+2 n+1}{3(n+1)^{2}}-\frac{2 n^{3}\left(n^{2}+2 n-1\right)}{\left(n^{2}+1\right)^{2}\left(n^{2}-1\right)}+\frac{n^{2}\left(n^{2}+1\right)}{\left(n^{2}-1\right)^{2}} \log n-\frac{n^{2}\left(n^{2}-1\right)^{2}}{\left(n^{2}+1\right)^{2}} \log \frac{n(n+1)}{n-1}
$$

The hypothesis of a nonabsorbing water layer is invalid in the SWIR. Bach and Mauser (1994)

calculate its transmittance $T_{w}$ with the Beer-Lambert law: $T_{w}=\exp \left(-\alpha_{B} L\right)$ with $\alpha_{B}$ the specific absorption coefficient of in situ water determined empirically $\left[\mathrm{m}^{-1}\right]$ and $L$ the thickness of the water layer $[\mathrm{m}]$. Therefore, the reflectance of a wet soil is written in the form

$$
R_{w s B}=\left(1-A_{w s L}\right) \exp \left(-2 \alpha_{B} L\right)
$$

with $A_{w s L}$ the total absorptance of a wet soil defined in Eq. (1). The factor 2 is because the light ray crosses twice the water layer. Considering that the soil surface may be a patchwork of wet and dry areas, Bach (1995) introduced an efficiency term $\varepsilon$ that accounts for the fraction of wet soil:

$$
R_{\text {modB }}=\varepsilon \times R_{w s B}+(1-\varepsilon) \times R_{d}
$$

$\varepsilon=0$ means that the soil is dry and $\varepsilon=1$ that it is covered with a film of water over its whole surface. 
In MARMIT the transmittance of light through the water layer is taking into account along the path

221 of the ray (Fig. 1). The expression for the total reflectance of the water/soil system can be derived by summing the amplitudes of successive reflections and refractions at the top of the water layer:

$$
R_{w s}=r_{12}+t_{12} T_{w}^{2} R_{d} t_{21}+t_{12} T_{w}^{4} R_{d}^{2} t_{21} r_{21}+\cdots
$$

Eq. (6) can be easily factorized in

$$
R_{w s}=r_{12}+t_{12} t_{21} R_{d} T_{w}^{2}\left(1+r_{21} R_{d} T_{w}^{2}+r_{21}^{2} R_{d}^{2} T_{w}^{4}+\cdots\right)
$$

The expression in brackets is a geometric series of general term $x=r_{21} R_{d} T_{w}^{2}$ that converges to $\frac{1}{1-x}$ if $|x|<1$. So we obtain

$$
R_{w s}=r_{12}+\frac{t_{12} t_{21} R_{d} T_{w}^{2}}{1-r_{21} R_{d} T_{w}^{2}}
$$

with $T_{w}=\exp (-\alpha L), \alpha$ the absorption coefficient of pure liquid water provided by Palmer and Williams (1974). If the soil is dry, the transmission-related parameters $t_{12}, t_{21}$ and $T_{w}$ are 1 and the reflections $r_{12}$ and $r_{21}$ are 0 , then $R_{w s}=R_{d}$. MARMIT also introduces an efficiency term $\varepsilon$ like in Eq. (5),

$$
R_{\text {mod }}=\varepsilon \times R_{w s}+(1-\varepsilon) \times R_{d}
$$

$$
\text { In the following, the term } r_{12} \text { is ignored because the diffuse radiation is negligible for our }
$$
laboratory measurements and none of the measurements described hereafter have been acquired in the specular direction while the water layer is assumed to be flat. In conclusion, MARMIT is physically more consistent than the previous models because the coupling between the multiple reflections and the absorption of light in the water layer is more realistic. The differences between the models presented above are summarized in Table 1.

\begin{tabular}{cccc}
\hline Author & Lekner and Dorf (1988) & Bach (1995) & MARMIT \\
\hline Physical variable & absorptance & reflectance & reflectance \\
\hline Expression & $A_{w s L}$ & $R_{w s B}$ & $R_{w s}$ \\
\hline
\end{tabular}




\begin{tabular}{cccc}
\hline Equation & $\frac{t_{12} A_{d}}{1-r_{21} R_{d}}$ & $\left(1-A_{w s L}\right) \exp \left(-2 \alpha_{B} L\right)$ & $\frac{t_{12} t_{21} R_{d} T_{w}^{2}}{1-r_{21} R_{d} T_{w}^{2}}$ \\
\hline \multirow{3}{*}{ Remark } & $\begin{array}{c}\text { Summation of the } \\
\text { absorptances above the } \\
\text { water layer; no light } \\
\text { absorption in the water } \\
\text { layer }\end{array}$ & $\begin{array}{c}\text { Transmittance of light in } \\
\text { the water layer accounted } \\
\text { for and multiplied by } \\
1-A_{w L D} ; \text { empirical } \\
\text { absorption coefficient of } \\
\text { water }\end{array}$ & $\begin{array}{c}\text { Transmittance of light in } \\
\text { the water layer directly } \\
\text { added in the path of the } \\
\text { ray; absorption coefficient } \\
\text { of pure liquid water }\end{array}$ \\
\hline
\end{tabular}

Table 1. Summary of every model described in this section.

\subsection{Datasets}

MARMIT was tested on a database gathering six datasets published in the literature, plus a new

one generated in the frame of this study (Table 2). They are, in order of publication year, Liu02 (Liu

et al., 2002, 2003), Lob02 (Lobell and Asner, 2002), Whit04 (Whiting, 2004; Whiting et al., 2004),

(Tian and Philpot, 2015a,b), and Bab16 (this article). They represent a total of 217 soil samples.

Ideally the number of SMC levels would cover the full range of soil moisture variation and textural

information about the soil samples would be available. The incompleteness of the datasets does not

allow completely validating MARMIT, analyzing its performance, and explaining the results. For

dataset includes enough SMC levels with relevant information on soils.

\begin{tabular}{cccccccc}
\hline Dataset & $\boldsymbol{N}$ & \multicolumn{2}{c}{$\boldsymbol{S M C}$} & Drying protocol & Sieving & $\boldsymbol{\theta}_{\boldsymbol{i}}$ & Bulk density \\
\cline { 3 - 4 } & & Number of levels & range & & & & \\
\hline Liu02 & 92 & 4 & $0-83 \%$ & Oven-dried & $2 \mathrm{~mm}$ & $15^{\circ}$ & $0.98-1.88$ \\
Lob02 & 4 & 9 to 15 & $0-118 \%$ & Oven-dried & $2 \mathrm{~mm}$ & $15^{\circ}$ & $0.64-1.54$ \\
Whit04 & 60 & 10 to 12 & $0-45 \%$ & Air-dried & $2 \mathrm{~mm}$ & & $0.88-1.36$ \\
Les08 & 32 & 6 & $0-87 \%$ & Oven-dried & no & $15^{\circ}$ & \\
Mar12 & 9 & 25 to 30 & $0-50 \%$ & Humidification & no & $25^{\circ}$ & \\
Phil14 & 3 & 97 to 205 & $0-45 \%$ & Air-dried & $2 \mathrm{~mm}$ & $30^{\circ}$ & $0.95-1.53$ \\
Bab16 & 17 & 6 to 8 & $0-40 \%$ & Oven-dried & $2 \mathrm{~mm}$ & $15^{\circ}$ & \\
\hline
\end{tabular}

Table 2. Summary of the main information on the datasets. $N$ is the number of soil samples, $S M C$ is the soil moisture content, $\theta_{i}$ is the angle of the incident light. 
to three textural soil classes: clay loam, sandy clay loam, and silty clay loam; those sampled in

241 Tomelloso also include three soil classes: loam, sandy loam, and silt loam. Organic matter content is

242 low in both regions $(<2 \%)$. Soil mineralogy $\left(\mathrm{CaCO}_{3}\right.$ and $\mathrm{SiO}_{2}$ contents $)$ is provided for four soils

243 from Tomelloso and five soils from Lemoore. Whiting et al. (2004) removed the reflectance between

$244760 \mathrm{~nm}$ and $950 \mathrm{~nm}$ because of detector artifacts in that region. The textural information is available

245 for the 92 soils of Liu02, the 32 soils of Les08, and 10 out of 17 soils of Bab16 (Fig. 2). Lob02

246 includes four soils displaying various mineralogical compositions, amounts of organic matter, and

247 porosities. Mar12 includes six different soils collected in the region of Reims (France). Three of them

248 (soils 1, 2 and 4) have one replicate.

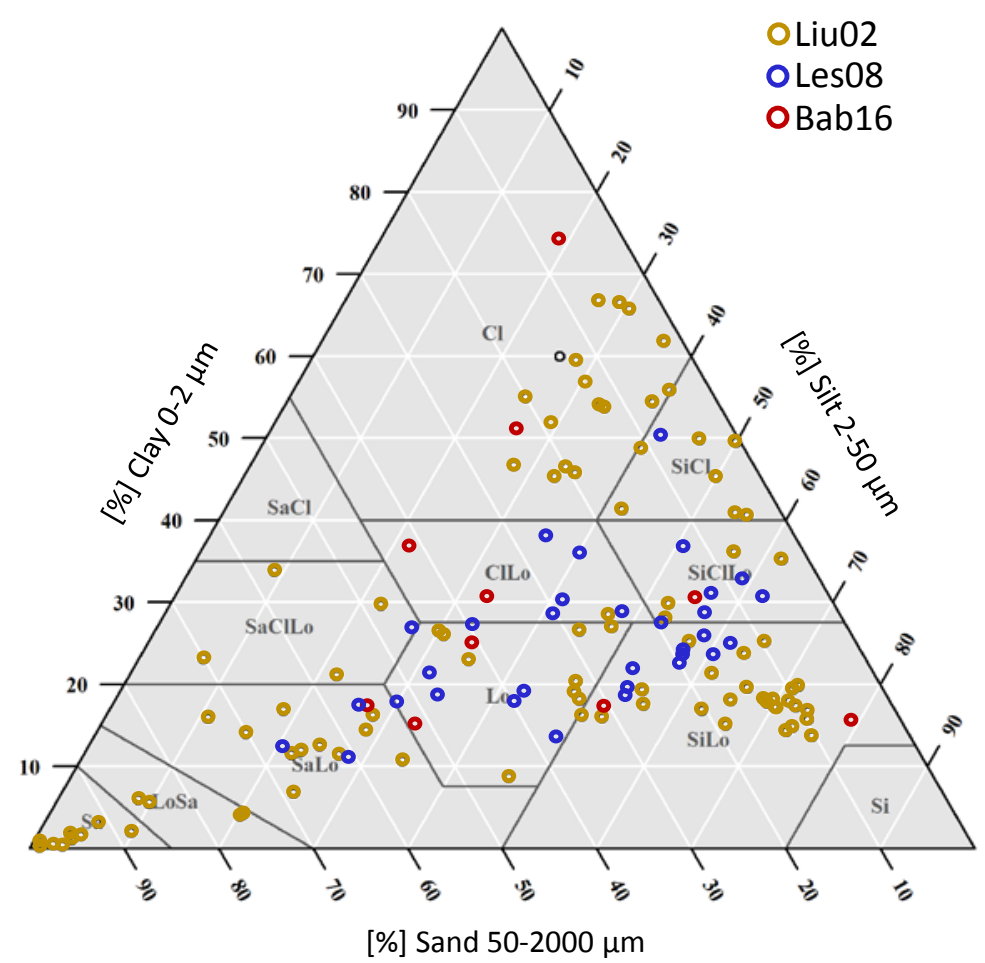

Fig. 2. Soil texture triangle showing the 12 major textural classes and particle size scales as defined by the USDA, for 134 soils of three datasets (Liu02, Les08, and Bab16).

Soil moisture content was most of the time expressed as a weight percent $\left(\mathrm{SMC}_{\mathrm{g}}\right)$ :

$$
S M C_{g}=\frac{M_{w s}-M_{d s}}{M_{d s}}=\frac{M_{H_{2} O}}{M_{d s}}
$$

with $M_{w s}$ [g] the mass of wet sample, $M_{d s}$ [g] the mass of dry sample, and $M_{H_{2} O}$ [g] the mass of 


$$
S M C_{v}=\frac{V_{w s}-V_{d s}}{V_{d s}}=\frac{V_{H_{2} O}}{V_{d s}}
$$

with $V_{w s}\left[\mathrm{~cm}^{3}\right]$ the volume of wet sample (dry soil and water-filled pores), $V_{d s}\left[\mathrm{~cm}^{3}\right]$ the volume of dry sample, and $V_{\mathrm{H}_{2} \mathrm{O}}\left[\mathrm{cm}^{3}\right]$ the volume of water. $\mathrm{SMC}_{\mathrm{g}}$ and $\mathrm{SMC}_{\mathrm{v}}$ are related by the dry bulk density of the sample (Lobell and Asner, 2002):

$$
S M C_{v}=\frac{V_{\mathrm{H}_{2} \mathrm{O}}}{V_{d s}}=\frac{M_{\mathrm{H}_{2} \mathrm{O}} \times d_{d s}}{M_{d s} \times d_{\mathrm{H}_{2} \mathrm{O}}}=S M C_{g} \times \frac{d_{d s}}{d_{\mathrm{H}_{2} \mathrm{O}}}
$$

with $d_{H_{2} O}$ [g.cm ${ }^{-3}$ the density of water $(\sim 1)$ and $d_{d s}\left[\right.$ g. $\left.\mathrm{cm}^{-3}\right]$ the dry bulk density of the sample.

Because densities of the soils were not available for every dataset, $\mathrm{SMC}_{\mathrm{g}}$ has been used in this study instead of $\mathrm{SMC}_{\mathrm{v}}$. In the following, if nothing is specified, the $\mathrm{SMC}$ will refer to the definition of $\mathrm{SMC}_{\mathrm{g}}$.

The experiments vary somewhat so we refer the reader to the original articles for spectrometer setup and moisture measurement protocol. Most of the data were acquired with an ASD FieldSpec spectroradiometer (Analytical Spectral Devices, Inc.) except for Whit04 where a Cary 5E spectrophotometer (SpectraLab Scientific, Inc.) was used. All the datasets but Mar12 were acquired according to the same protocol: the soil sample is sieved, put into a Petri dish with a radius of about 5 cm, moistened up to saturation (except for Liu02 where the saturation stage is exceeded) and then dried. At regular intervals during drying, the soil samples are weighed in order to assess SMC and their reflectance spectrum is recorded. Mar12 was obtained in a somewhat different way since the soil samples were not sieved and moistened after drying. Moreover, they were put into pie plates with a radius around $25 \mathrm{~cm}$.

All the datasets associate a reflectance spectrum with a water content value measured concomitantly. Since reflectance varies with the angle of the incident light $\theta_{i}$, precautions will be taken when comparing Mar12 and Phil 14 to the other datasets. Fig. 3 shows the evolution, as a function of water content, of the reflectance of a clay soil, a loamy soil, and a sandy soil picked at the vertices of the texture triangle. 

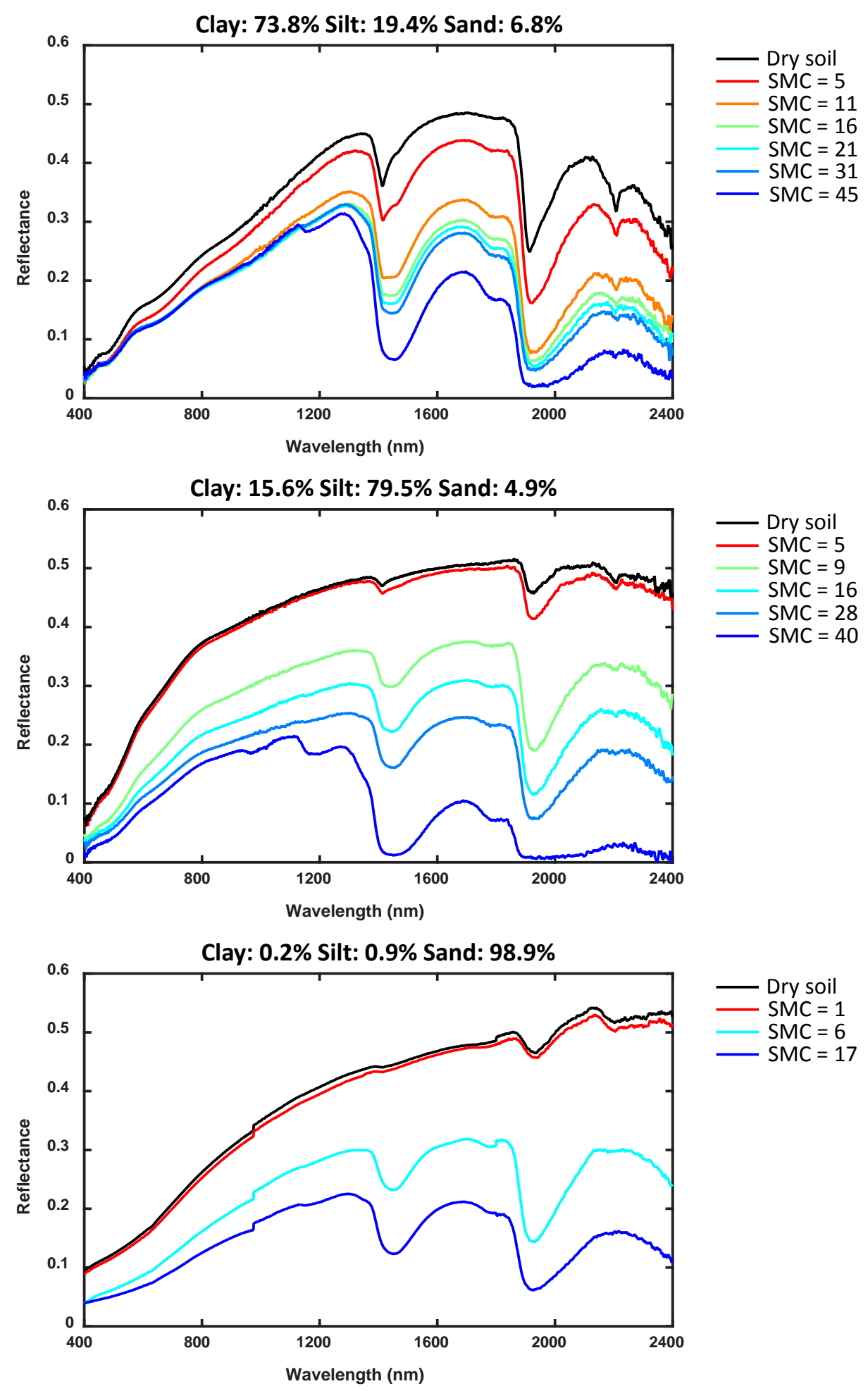

Fig. 3. Reflectance spectra and their associated $\mathrm{SMC}_{\mathrm{g}}$ (g/g). (top) Clay soil 724 Lebna57 in Bab16, (middle) loamy soil Luvisol 208Te in Bab16 and (bottom) sandy soil 76 in Liu02.

The clay soil made of very fine particles holds greater amounts of water and has greater porosity than coarser soils, so the SMC at saturation is higher (Fig. 3, top). Conversely, the sandy soil made of large particles has a lower porosity; it rapidly reaches saturation (Fig. 3, bottom). Finally, the SMC of the loamy soil at saturation is in-between (Fig. 3, middle). 


\subsection{The MARMITforSMC approach}

The method for retrieving soil moisture content with MARMIT called MARMITforSMC involves

290

291

292

293

294

295

296

297

298

299

300

301

302

three steps (Fig. 4):

(1) Inversion step: the water thickness $L$ and the efficiency $\varepsilon$ are estimated by model inversion (Eq. (14)). One can note that the reflectance of the dry soil is needed to infer the reflectance of a wet soil.

(2) Calibration step: a statistical relationship is established between the mean water thickness (mean light path) defined as $\varphi=L \times \varepsilon$ and the measured SMC. Here we use $\varphi$ instead of $L$ because, for very small SMC, $\varepsilon$ changes slowly in comparison to $L$ which can vary very quickly.

(3) Assessment step: SMC is retrieved by applying the relation found in the calibration step and compared with the measured values.

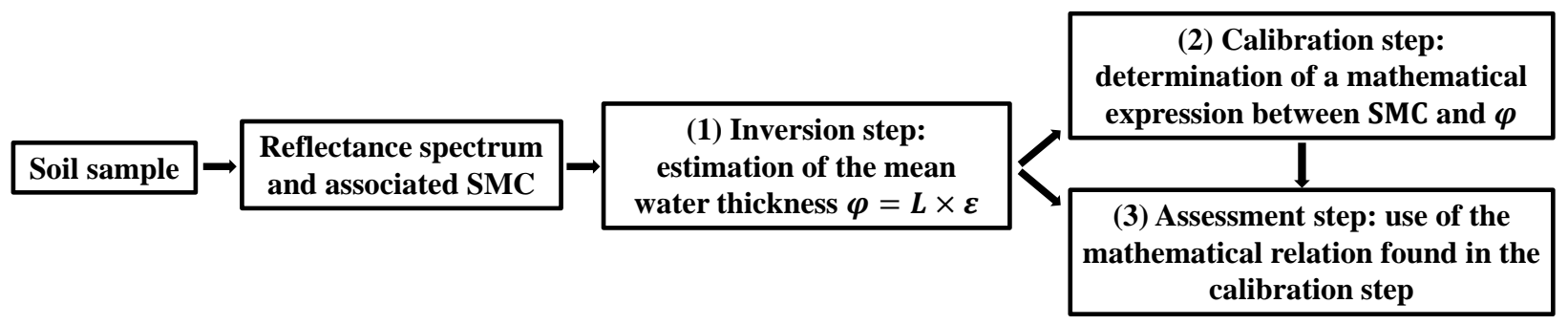

Fig. 4. Flowchart representing the overall MARMITforSMC method used in this study.

\section{Results}

The root-mean-square error (RMSE) that measures the difference between the measured and estimated values of SMC was chosen to compare the efficiency of the various regressions and models:

$$
R M S E=\sqrt{\frac{\sum_{i=1}^{N}\left(S M C_{i}^{\text {meas }}-S M C_{i}^{e s t}\right)^{2}}{N}}
$$

where $S M C_{i}^{e s t}$ is the estimated SMC for the soil sample $i, S M C_{i}^{\text {meas }}$ is the measured SMC, and $N$ is the number of SMC. 


\subsection{Inversion step}

311 The inversion step is applied to all the samples. Model fitting is achieved by searching the best

312 values of $L$ and $\varepsilon$ that minimize the merit function $\chi^{2}$ thanks to the bounded simplex search algorithm, 313 implemented in MATLAB (Nelder and Mead, 1965):

314

315

$$
\chi^{2}(L, \varepsilon)=\sqrt{\frac{\sum_{\lambda_{1}}^{\lambda_{2}}\left(R_{\text {meas }}(\lambda)-R_{\text {mod }}(\lambda, L, \varepsilon)\right)^{2}}{n_{\lambda}}}
$$

316 with $R_{\text {meas }}$ the measured reflectance at wavelength $\lambda, R_{\text {mod }}$ the modeled reflectance described in Eq.

317 (9) at wavelength $\lambda$, and $n_{\lambda}$ the number of wavelengths. In our case, $\lambda_{1}=400 \mathrm{~nm}$ and $\lambda_{2}=2400 \mathrm{~nm}$.

318 The fit is good for most of the soils $\left(r^{2}>0.95\right.$, Fig. 5, top) but, when SMC is higher than $20 \%$, the 319 reflectance of some soils is overestimated by the model outside the water absorption bands and 320 underestimated within these bands ( $r^{2}<0.90$, Fig. 5, bottom). This misfit decreases if $\varepsilon$ is 321 unconstrained but we bounded it between 0 and 1 to keep a physical meaning.

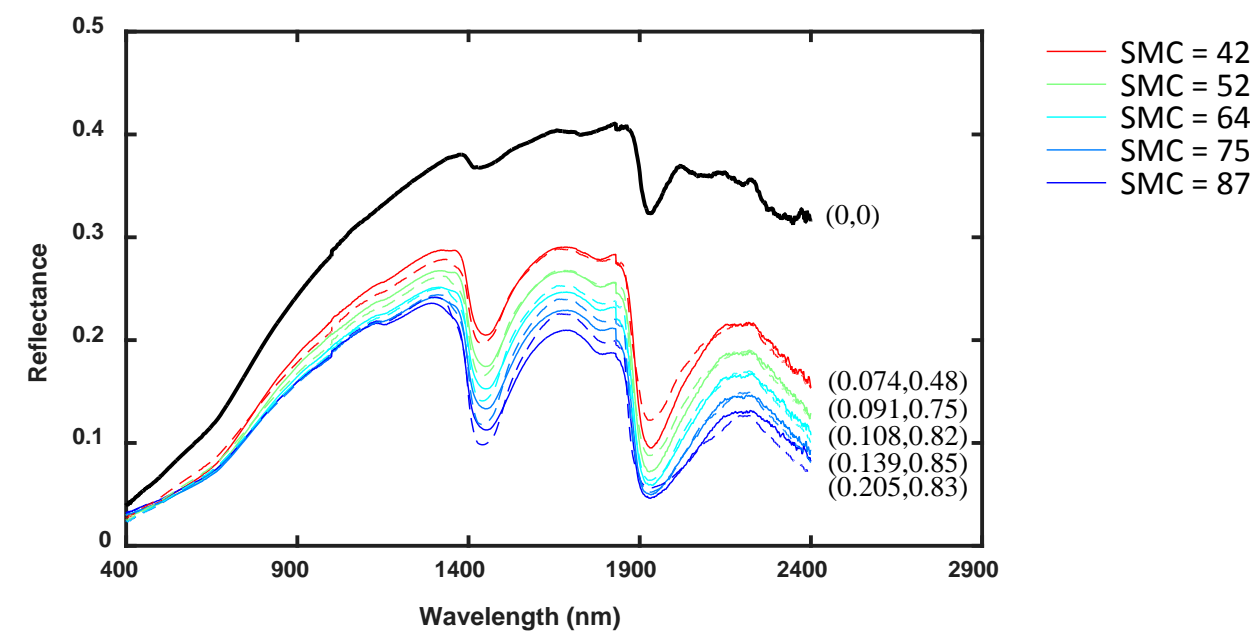



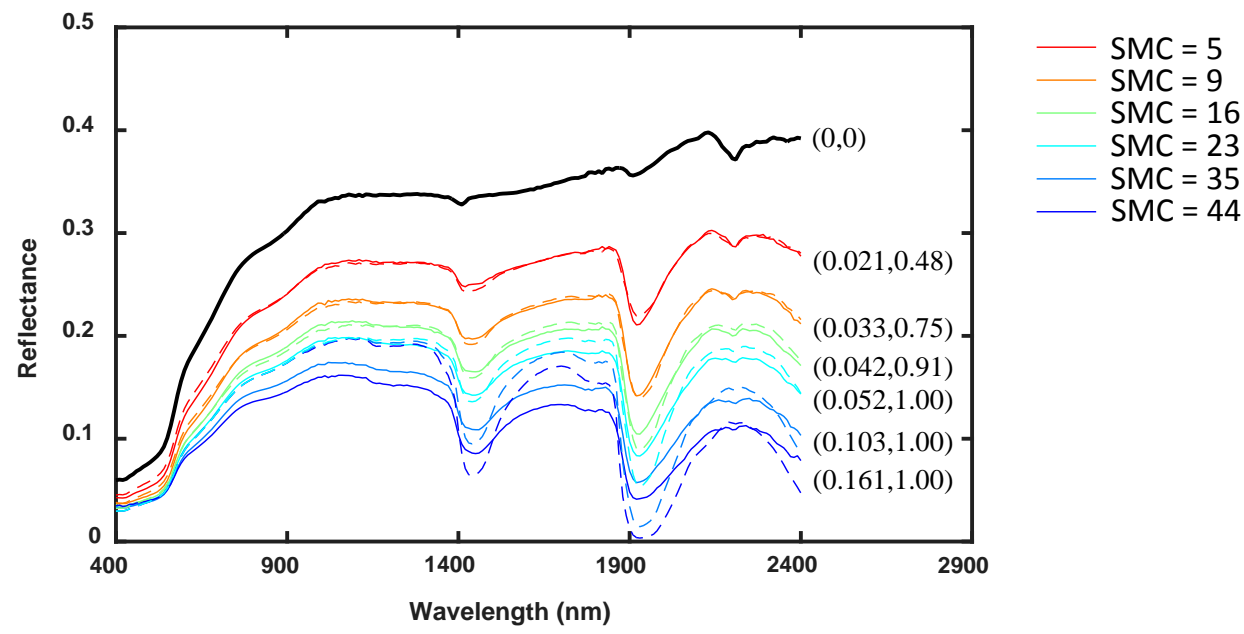

Fig. 5. Measured (solid line) and modeled (dashed line) reflectance spectra of two soils at various levels of soil water content: (top) 30PrairieB in Les08 with SMC in weight percent (g. $\mathrm{g}^{-1} \times 100$ ) and (bottom) Entisol in Lob02 with SMC in volumetric percent $\left(\mathrm{cm}^{3} . \mathrm{cm}^{-3} \times 100\right)$. The retrieved parameters of the model are provided to the right of the curves $(L, \varepsilon)$. $L$ is in $\mathrm{mm}$.

The RMSE between the measured and the modeled reflectance spectra of all soils of all dataset is generally lower than 2\% except at $1900 \mathrm{~nm}$, a major water absorption band (Fig. 6). Philpot (2010)

suspects that part of this discrepancy is due to a change in the optical properties of the liquid phase of

soil when water, that already contains dissolved organic matter and ions, is mixed with suspended absorption coefficient of pure liquid water (Fig. 6).

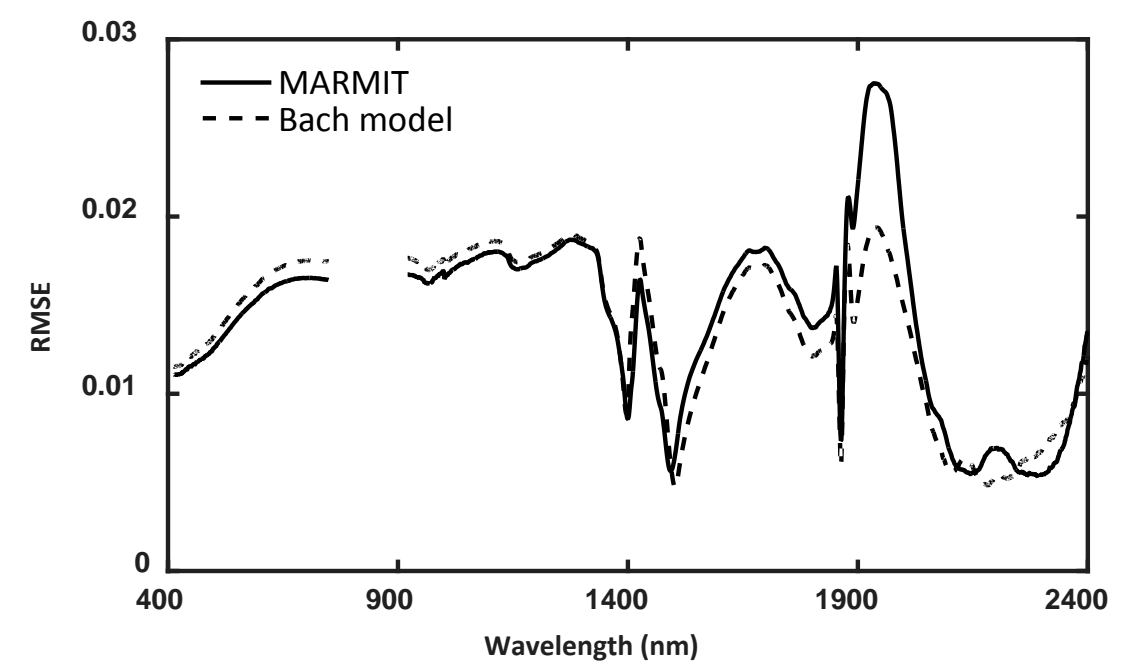

Fig. 6. Root-mean-square error (RMSE) between the soil spectra and two soil reflectance models, calculated for all the datasets. 


\subsection{Calibration and prediction steps}

The calibration step consists in finding a mathematical expression relating the mean water

thickness $(\varphi)$ to the soil moisture content (SMC). It is not possible to determine a unique relationship

valid for all soils of all datasets because the points are widely scattered (Fig. 7). The relationship

seems to depend on soil characteristics and measurement protocols: as an example the two white

quartz sands in Phil14 and Bab16, which are very bright, are distinct from the other soils. One also

notes that the soil samples of Mar12, which were wetted instead of being dried, display a relationship

shifted by about $0.1 \mathrm{~mm}$ on the right compared to the other soils. Tian et al. (2015b) mention that

water tends to stagnate on the surface of the soil during humidification so that a small amount of water

induces a significant change in reflectance. Conversely during drying, water and air are distributed

throughout the sample for most of the drying period, so that soil reflectance does not change much when the sample is almost dry.

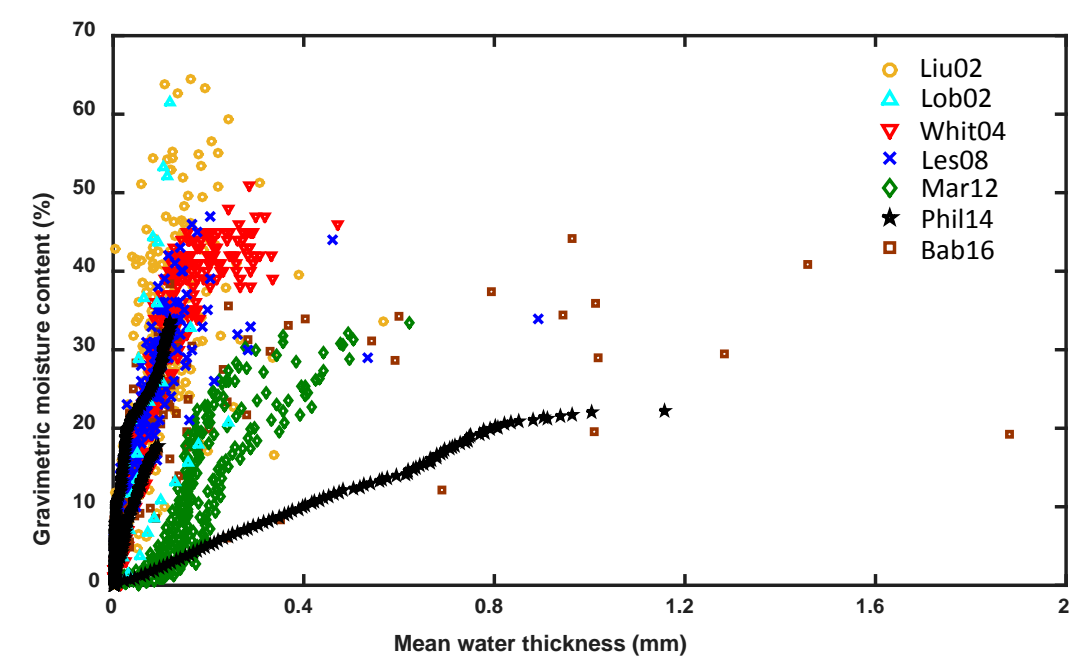

Fig. 7. Relationship between $\mathrm{SMC}_{\mathrm{g}}$ and $\varphi$ for all soils of all datasets.

Because no general relationship could be found, the soils were first studied separately and then gathered into classes based on textural, mineralogical, and spectral properties.

\subsubsection{Soil-by-soil calibration and prediction steps}

Several models such as polynomials of degree one (Bach and Mauser, 1994; Bach, 1995), two or 
353 three, as well as power functions have been tested to adjust the points relating SMC to $\varphi$. The S-shape

354 curvatures observed for almost every soil suggest using a logistic function:

$$
S M C=\frac{K}{1+a e^{-\psi \varphi}}
$$

357 with $K$ the maximum value of the curve, $\psi$ the steepness of the curve, and $a$ a translation factor that 358 moves the whole curve along the $\mathrm{x}$-axis. A simple one-factor-at-a-time sensitivity analysis of the 359 logistic function illustrates the effect of each parameter on the curve shape (Fig. 8). An increase in $K$ 360 induces an increase in the asymptote of the curve; an increase in $a$ shifts the whole curve to the right; 361 an increase in $\psi$ increases the slope of the curve and shifts the inflexion point towards the smallest 362 values of $\varphi$.
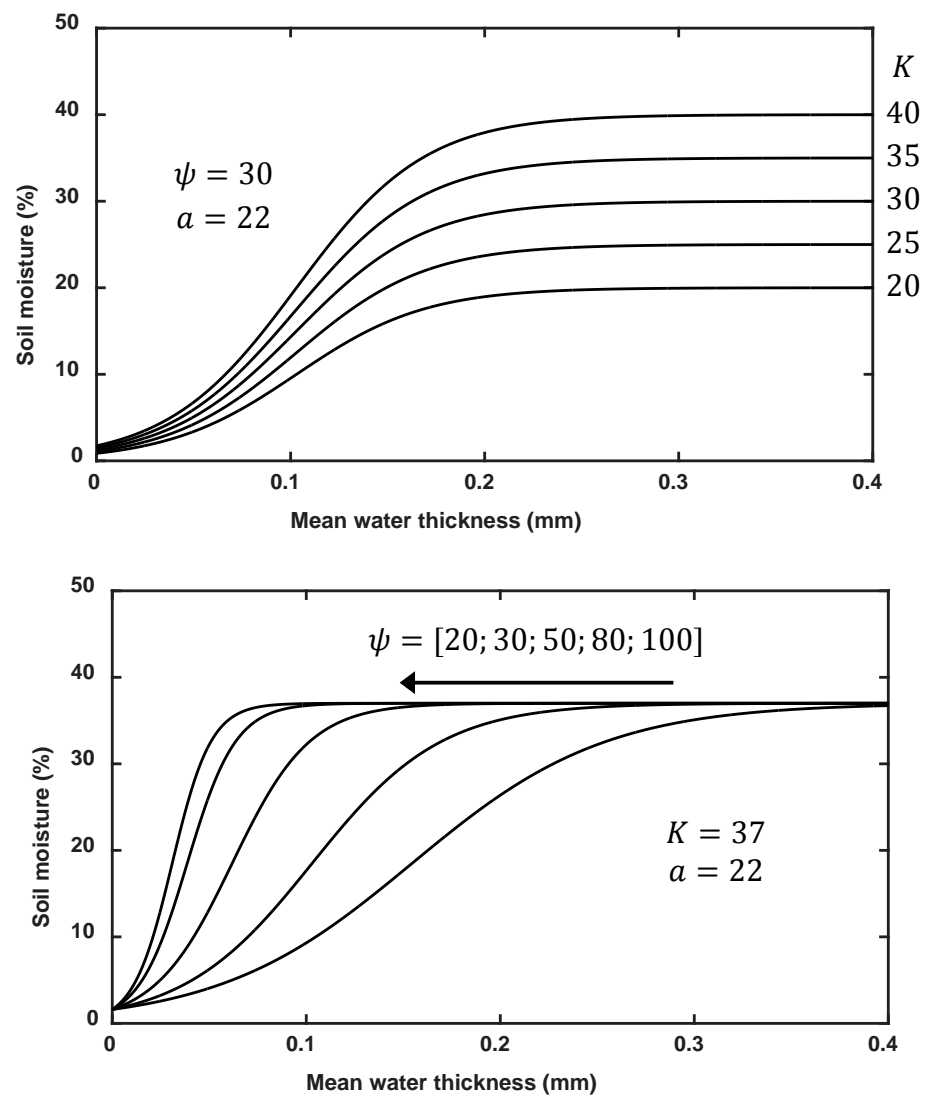


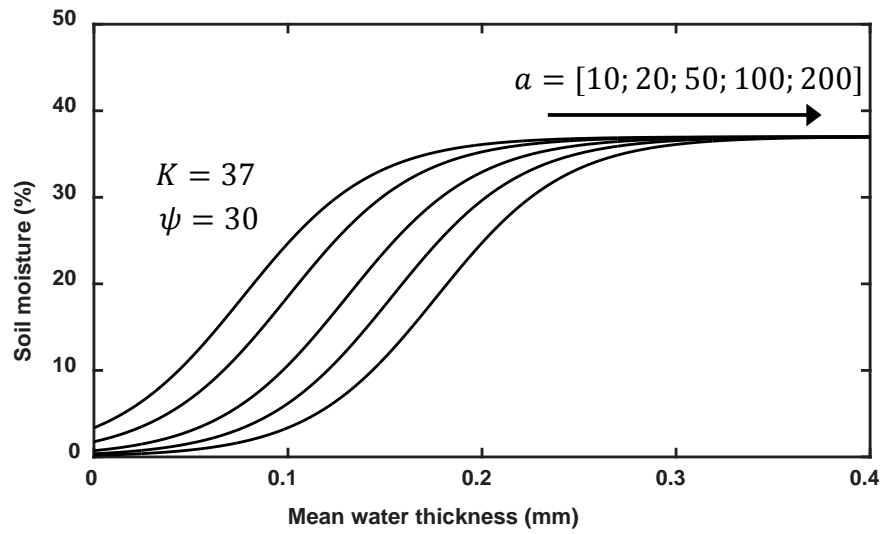

Fig. 8. Schematic trends of a logistic function. (top) $K$ is variable, $\psi$ and $a$ are constant; (middle) $\psi$ is variable, $K$ and $a$ are constant; and (bottom) $a$ is variable, $K$ and $\psi$ are constant.

Fig. 9 shows that the logistic function explains very well the evolution of the SMC with $\varphi$ for
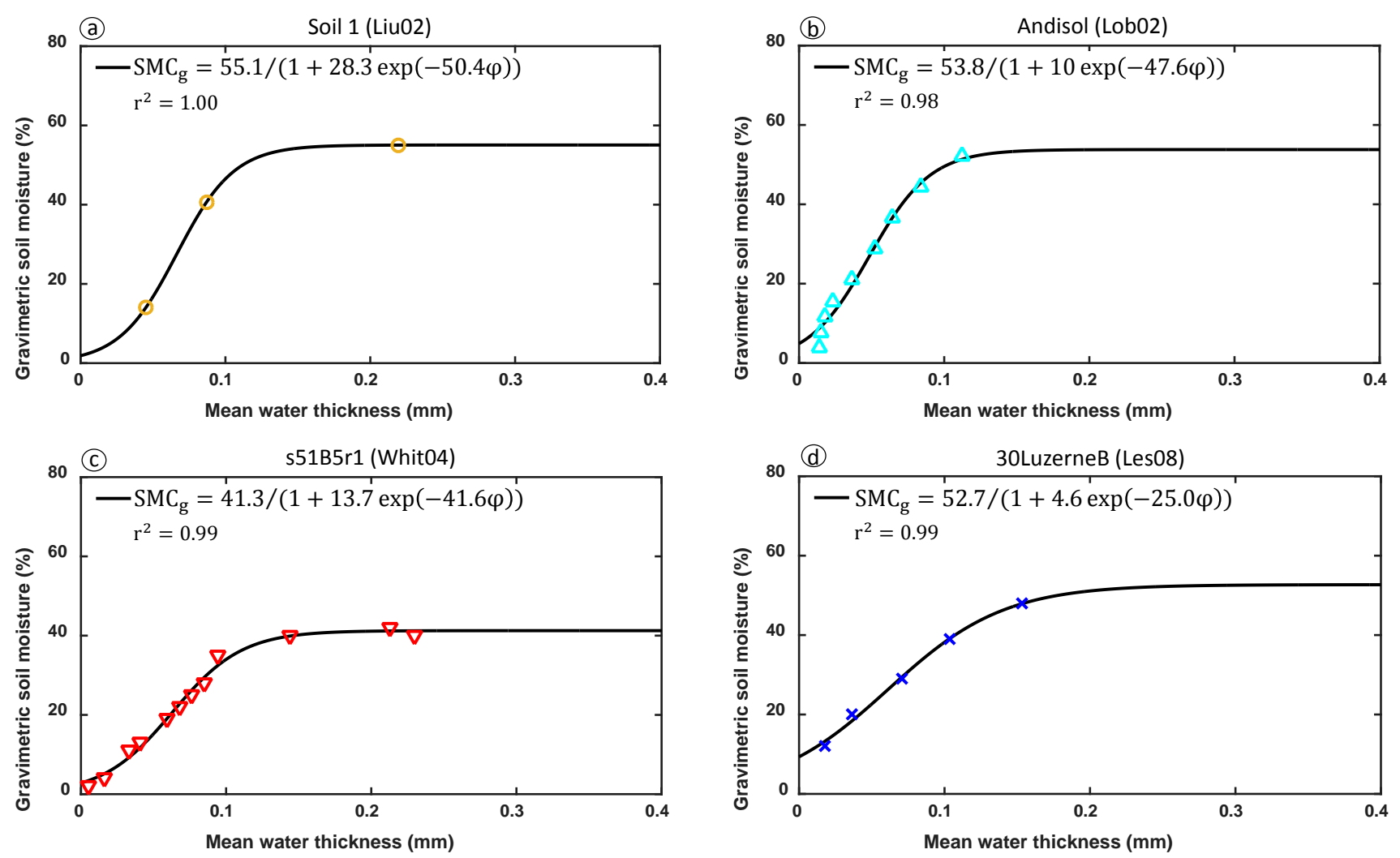

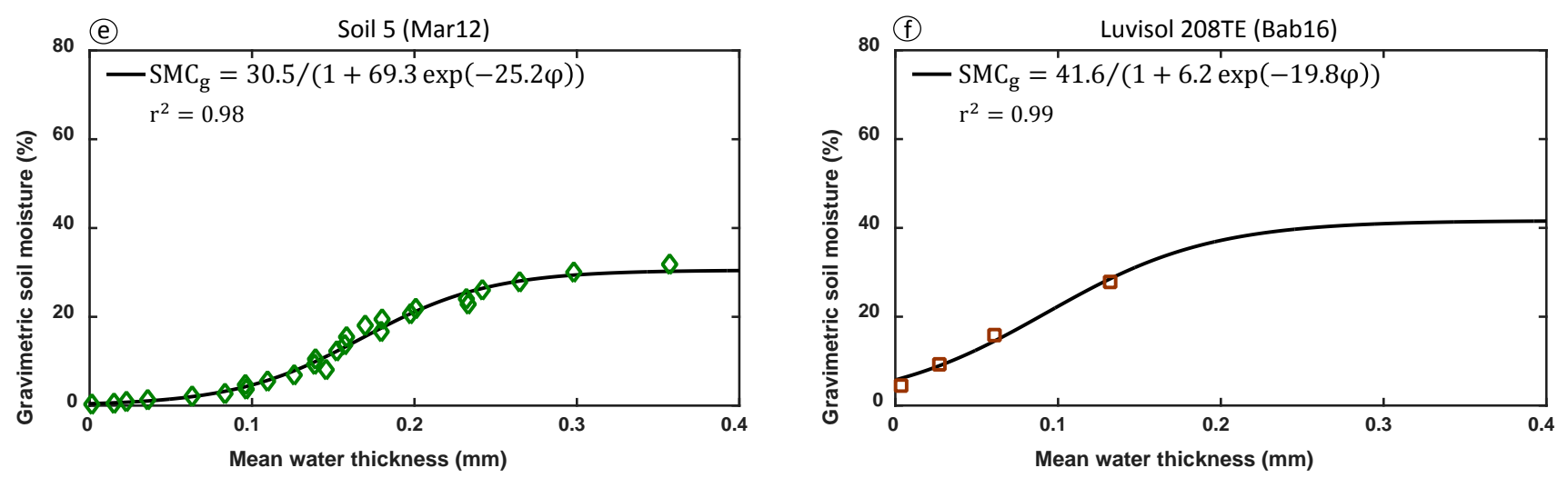

Fig. 9. Calibration step for six soils of six different datasets. Name of the soil in title and dataset in bracket.

Note that when $K$ is at the maximum value of SMC, i.e., saturation, the soil pores are filled with water and the volumetric water content is nearly equal to the porosity. Since the volumetric and the gravimetric water contents can be calculated by Eq. (12), $K$ may be related to the porosity of the soil. To validate this assumption, we plotted the maximum soil water content, i.e., the water content at saturation (SMCs), as a function of $K$ (Fig. 10). In most cases, there is a correspondence between the two but $K$ sometimes diverges from the bisector because of the limited range of soil moistures available in some datasets. If now we remove the soil samples containing less than six data points (twice the number of parameters in the sigmoid function), these two parameters are almost equivalent.
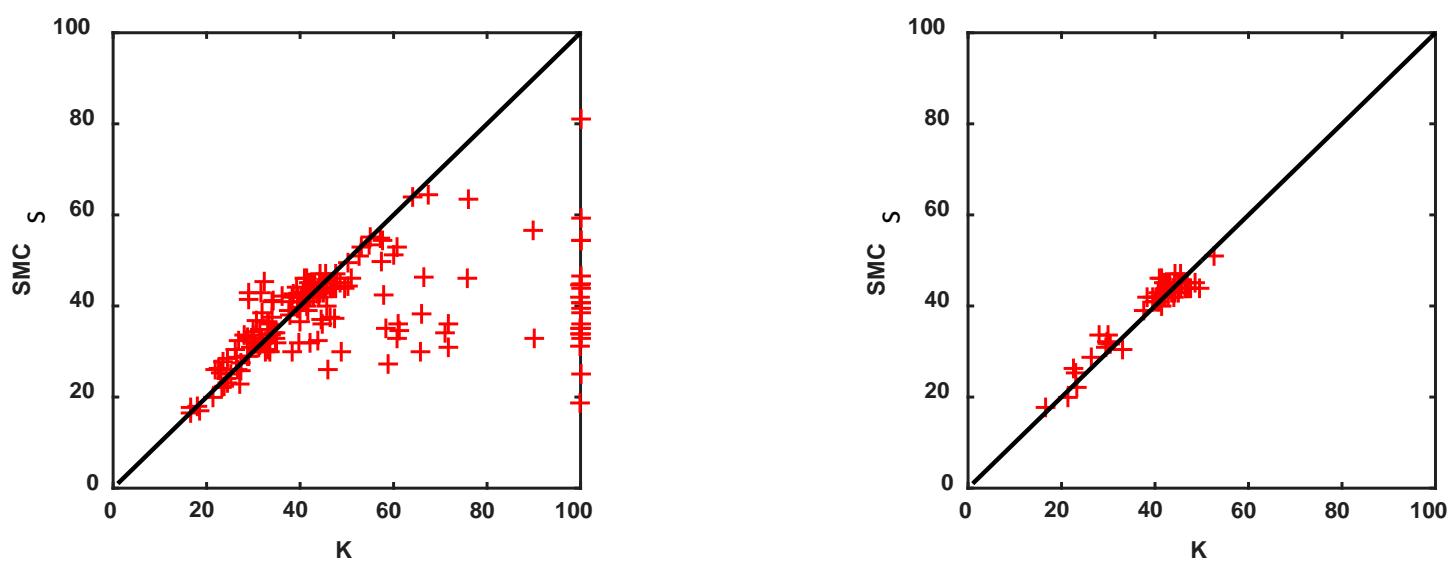

Fig. 10. Link between $K$ and SMCs (left) for every soil of every dataset and (right) for the soils with more than six moisture content measurements.

If the values of $\varphi$ determined during the inversion step are reinjected into the relationship found between SMC and $\varphi$, and if the measured and estimated soil moisture contents are compared, then 

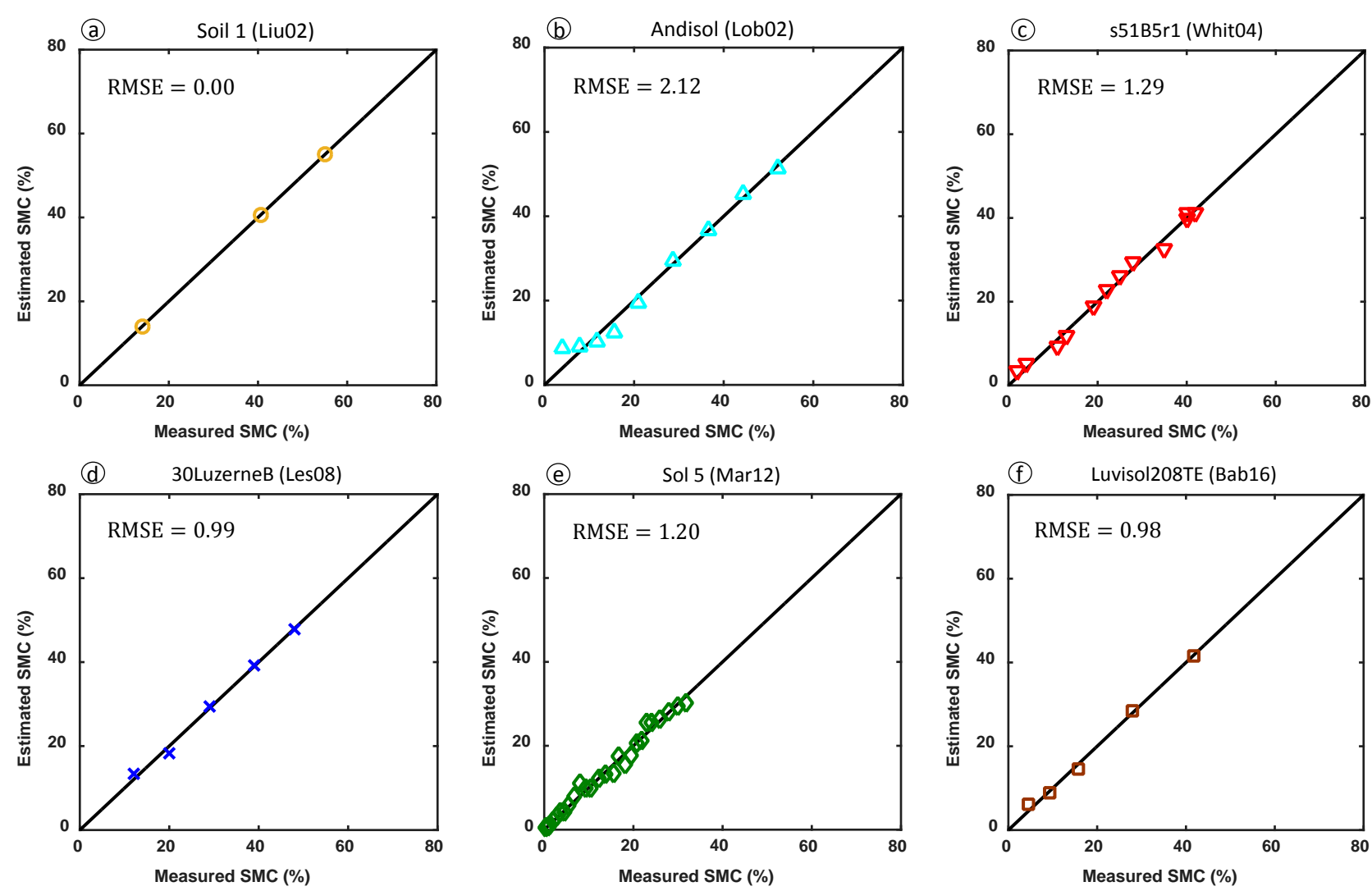

Fig. 11. Estimated vs measured SMC for the six soil samples used in the calibration step in the Fig. 9.

We used the replicates of the Whit04 dataset to test the soil-by-soil calibration and to validate the

method. A relationship has been adjusted between $\varphi$ and SMC for thirty soil samples, leading to as

many calibration equations that have been applied to the replicates. The estimation error is generally

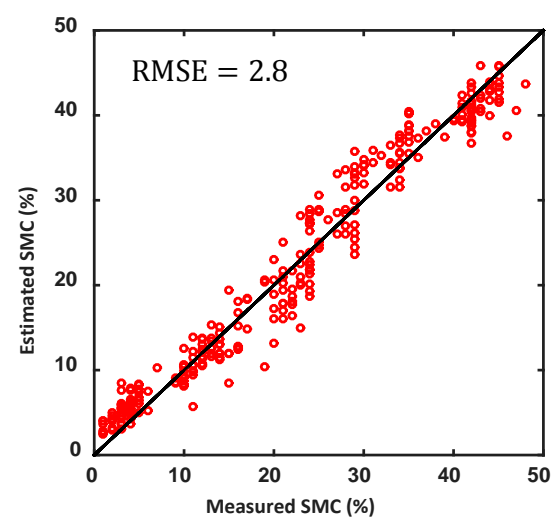

Fig. 12. Estimated vs measured SMC for the soils of the Whith04 dataset. 
One of the advantages of remote sensing is a large spatial coverage, but detailed knowledge of the

nature of the investigated soils is barely available because they may change quickly in terms of texture and structure from one spot to another. Therefore, an approach requiring a calibration relation between SMC and $\varphi$ for each soil hardly applies since there is no unique relation as shown in Fig. 7. In order to fulfill operational requirements, we attempted classifying soils into groups. Several techniques were used, based on soil physical, chemical and spectral characteristics.

\subsubsection{Class-by-class calibration and assessment steps: physical and chemical properties}

First, the effects of mineralogy and texture in the estimation of the relationship between SMC and $\varphi$ were investigated. To this end, we took advantage of the mineralogical information of the Whit04 dataset. The amount of calcium carbonate $\left(\mathrm{CaCO}_{3}\right)$ and silicon dioxide $\left(\mathrm{SiO}_{2}\right)$ in the Tomelloso soils is quite variable, while there is no $\mathrm{CaCO}_{3}$ and a constant amount of $\mathrm{SiO}_{2}(\sim 29 \%)$ in the Lemoore soils. The relationship between SMC and $\varphi$ is consequently more scattered for the first $\left(r^{2}=0.93\right.$ ) (Fig. 13a) than for the second $\left(r^{2}=0.97\right)$ (Fig. 13b). While the modification of the spectral properties of a soil when moistened depends upon its mineralogical composition (Bedidi et al., 1992), it may influence the parameters $\psi$ and $a$ of the sigmoid function. The soils of Les08 and Bab16 that contain textural information were also investigated. We divided them into two groups: sandy soils for which $50 \%$ of the grain size is sand (larger than $0.05 \mathrm{~mm}$ and less than $2 \mathrm{~mm}$ ) and clay soils for which $50 \%$ of the grain size is clay (less than $2 \mu \mathrm{m}$ ). The relationship between SMC and $\varphi$ is well fitted by the sigmoid function in both cases, with high coefficients of determination (Figs. 13c and 13d). Furthermore, the parameter $K$ is lower for the sandy soils (46.2\%) than for the clay and silt soils (59.1\%) in accordance with the fact that the porosity of the former is lower than that of the latter. This is consistent with the hypothesis that the parameter $K$ is related to this soil property. These results show that our method can be generalized by making a preliminary soil texture and mineralogical composition classification. Unfortunately, such information is seldom available. 

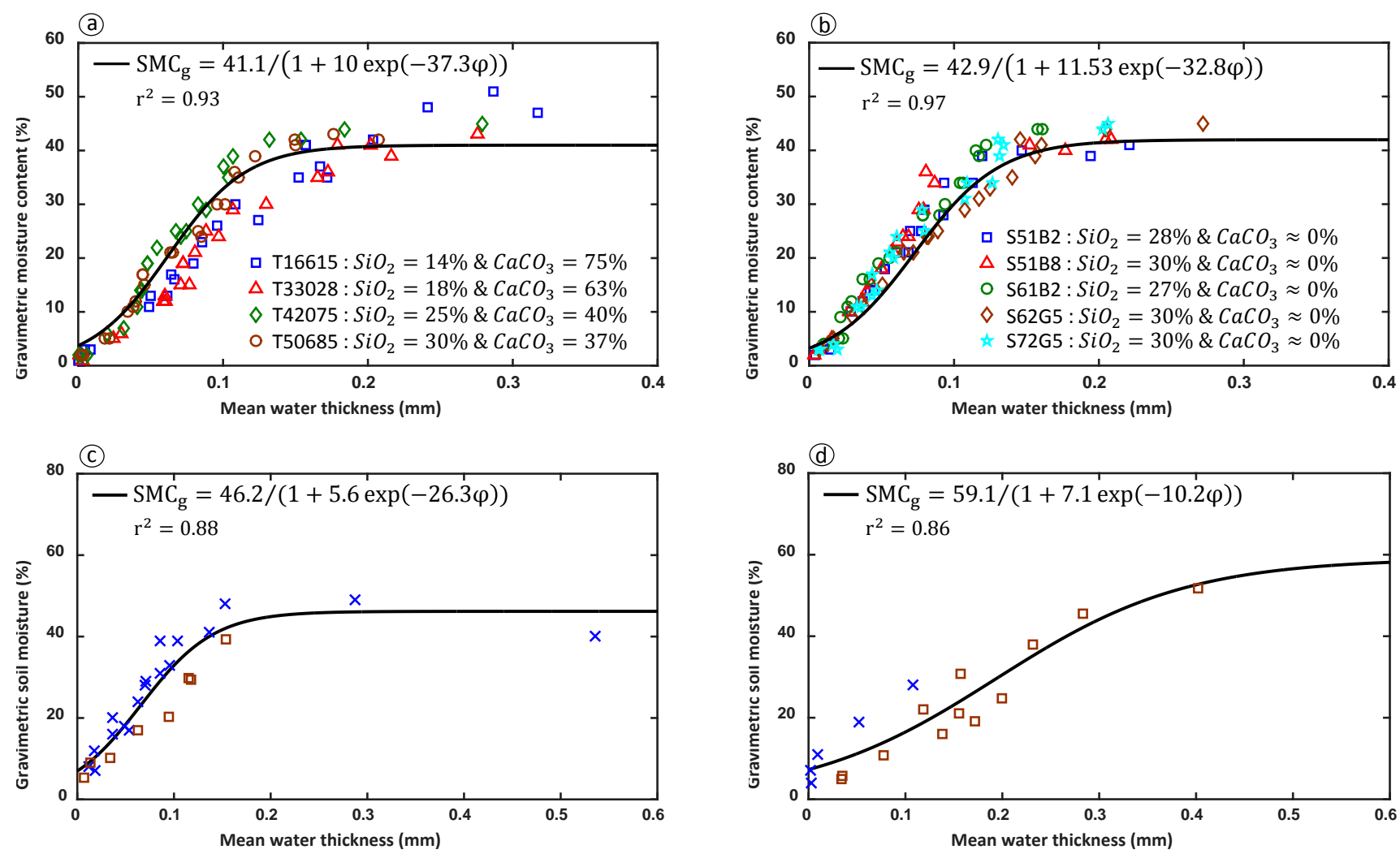

Fig. 13. Calibration phase for (a) four soils from Tomelloso and (b) five soils from Lemoore (Whit04 dataset). Calibration phase for (c) six sandy soils and (d) nine non-sandy soils of the Les08 (blue crosses) and Bab16 (brown squares) datasets.

\subsubsection{Class-by-class calibration and prediction: spectral signatures}

We investigated the classification method proposed by Lesaignoux et al. (2013) where the soils are

grouped together on the basis of the shape of the dry soil reflectance spectrum in four wavelength

ranges (VIS, NIR-SWIR, MWIR, and LWIR). The 32 soils were initially divided into nine groups but,

in this study, groups 1 and 2 and groups 4 and 5 were merged together because the shape of their dry

soil is identical in the VIS and NIR-SWIR and because our study is restricted to these domains.

Moreover groups 3, 7, 8 and 9 which contain less than two soils were discarded. Finally we

maintained three classes: class I (groups 1 and 2), class II (groups 4 and 5), and class III (group 6).

Figs. 14a-d compare the sigmoid functions obtained on these three classes and on the whole dataset.

Note that because some soils of Les08 are excluded from the three classes, some points of Fig. 14a do

not appear in Figs. 14b-d. The $r^{2}$ values of $0.71,0.84$ and 0.98 for class I, II and III, respectively, are

globally better than that of the whole dataset $\left(r^{2}=0.75\right)$. The calibration equation of class III

displays a very high coefficient of determination probably because all the samples have been collected 
have been divided into two sub-classes: one for the calibration and one for the validation (Figs. 14e-f).

436

437

438

439

440
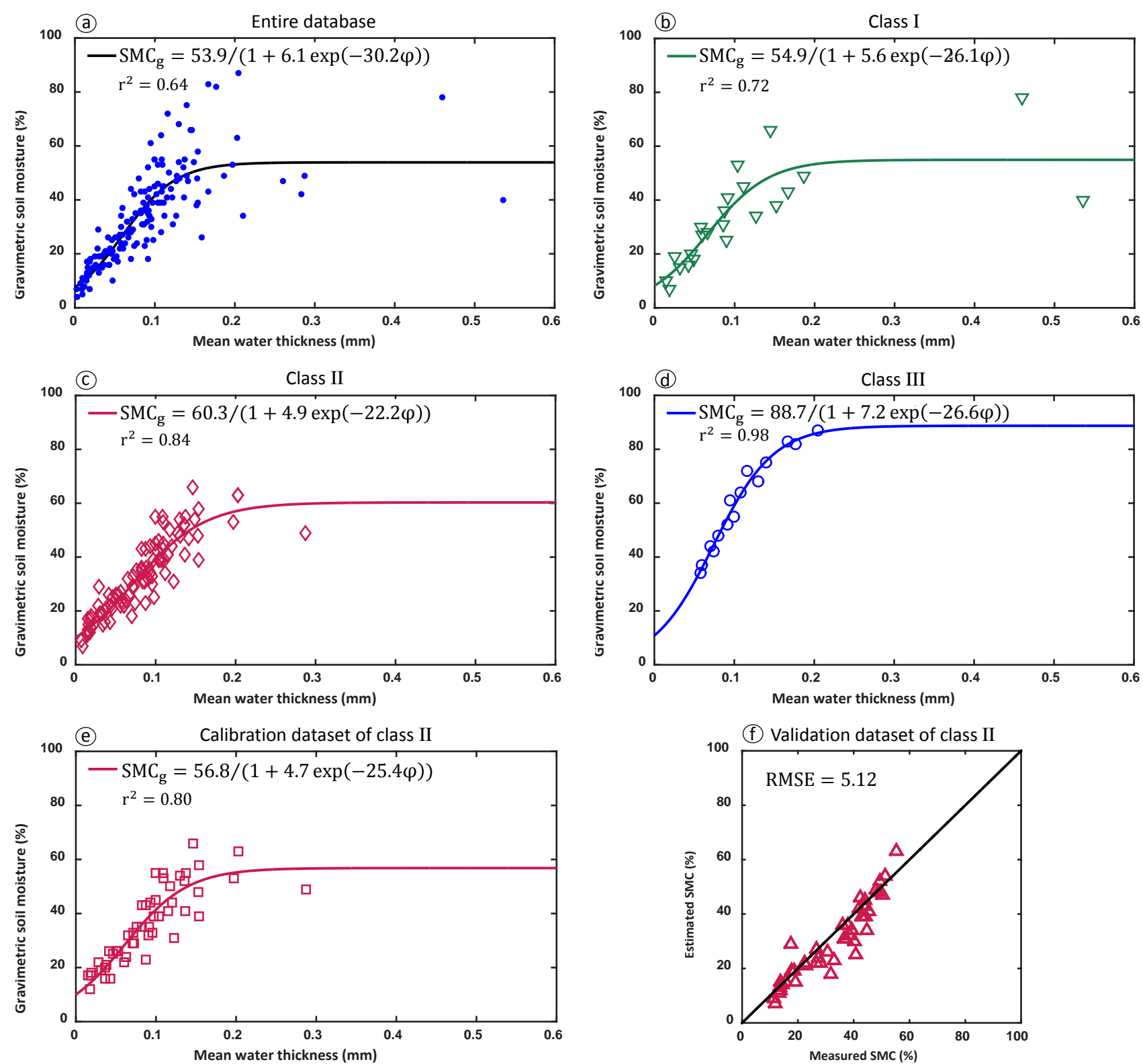
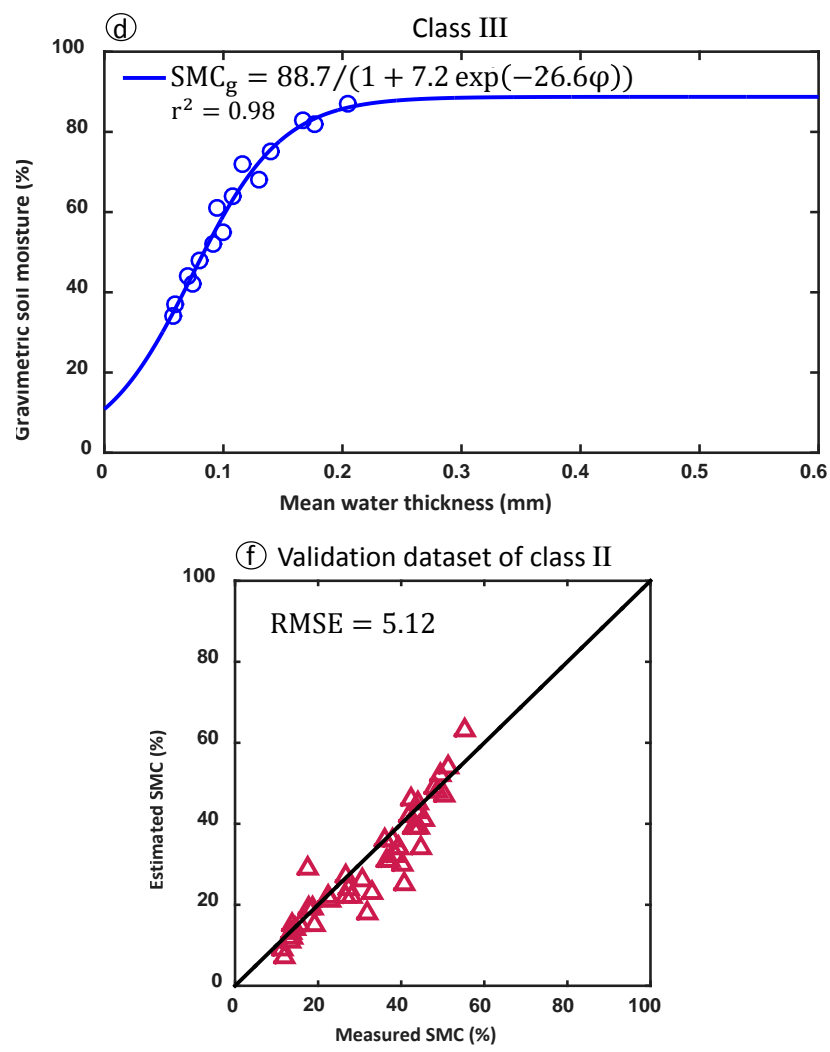

Fig. 14. Calibration phase for the different $a$ priori classes of the Les08 dataset: (a) the thirty-two soils of the whole dataset, (b) the five soils of class I, (c) the sixteen soils of class II and (d) the three soils of class III. (e) Link between SMC and $\varphi$ obtained with five soils of the class II and (f) estimated SMC thanks to the equation of calibration of (e) compared to measured SMC for the five soils of the validation dataset of class II.

Another classification method has been tested: Lacerda et al (2016) compare the reflectance of dry 
444 function of the first, clayey soils and sandy soils are respectively located at the bottom and the top of 445 the plot. The reflectance of sandy soils is often higher because they are often made of quartz (e.g., 446 Stoner and Baumgardner, 1981; Demattê, 2002; Lacerda et al., 2016). The Whit04 dataset that 447 contains two different kinds of soils is well suited to test this method. Fig. 15a shows that the 448 Tomelloso soils are located at the top of the plot and are scattered, while the Lemoore soils are located 449 at the bottom and are concentrated. The calibration performed on the whole dataset globally led to a 450 lower coefficient of determination $\left(r^{2}=0.94\right)$ than that performed on the Lemoore and Tomelloso 451 soils ( $r^{2}=0.96$ and $r^{2}=0.94$, respectively) (Figs. 15c-d). Therefore, by using the same method of 452 classification, we divided the Tomelloso soils into two subsets (Fig. 15b). The fit is slightly better 453 with coefficients of determination higher than 0.95 and the two classes are clearly separated (Fig. 15e). Once again the parameters of the sigmoid function, $\psi$ and $a$, seem to be related to the mineralogy of the soils, which corroborates the result of Figs. 13a-b.
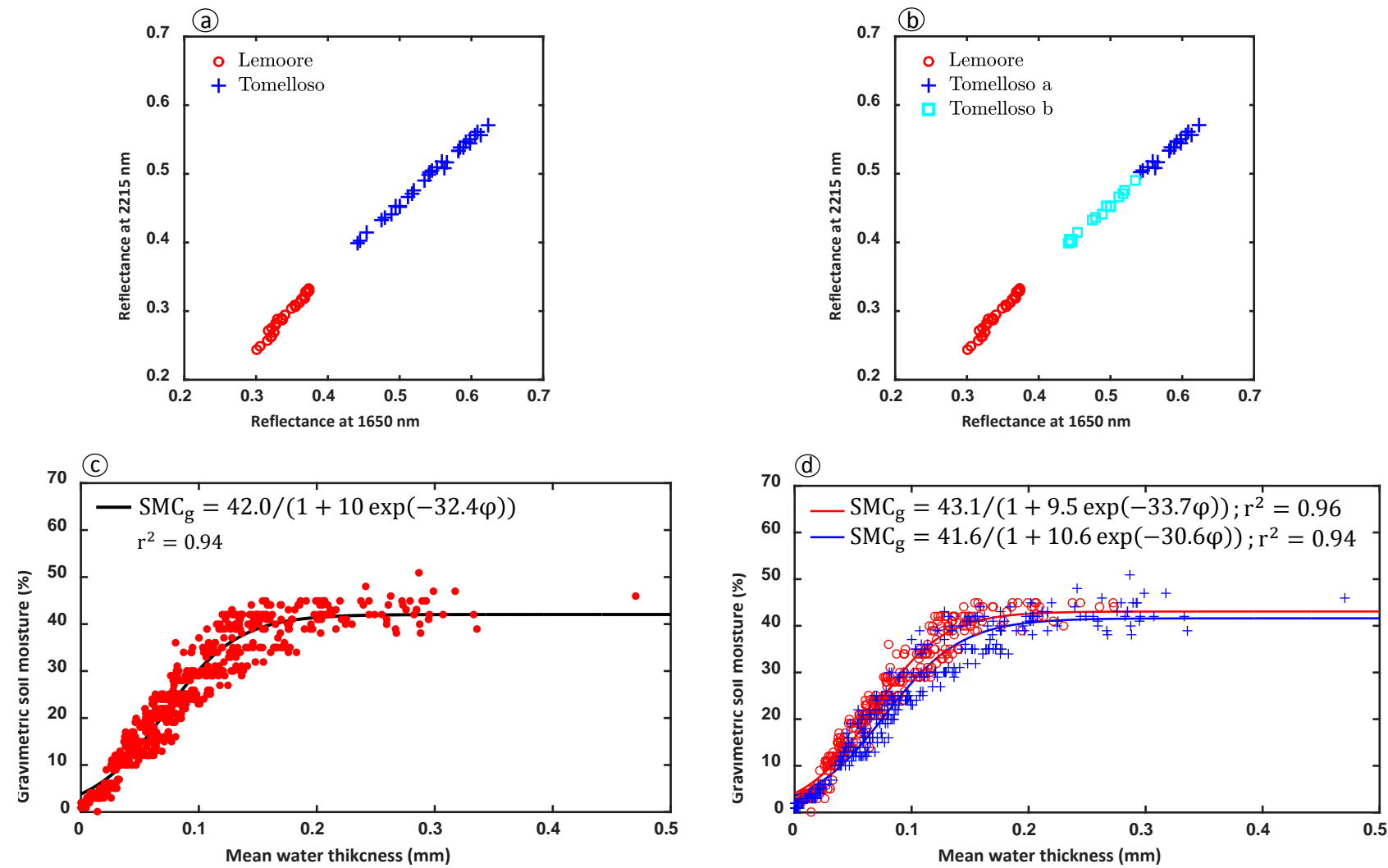


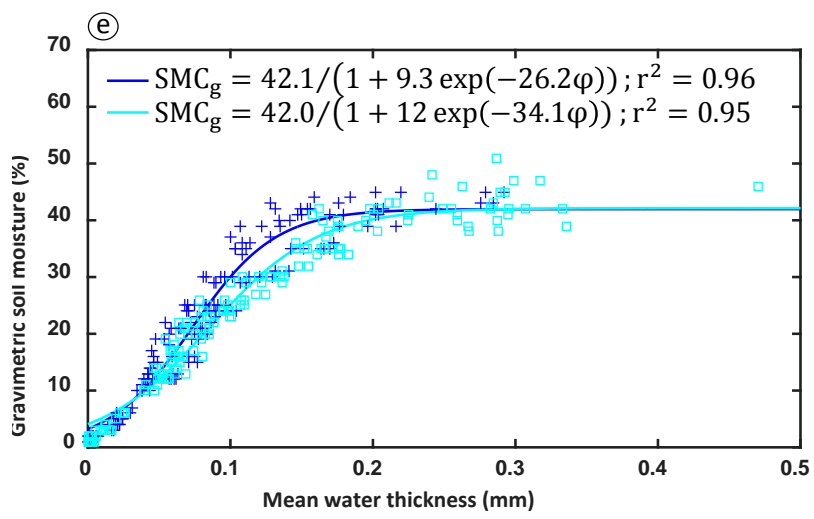

Fig. 15. Classification of the sixty soils of Whit04 after Lacerda et al. (2016). (a) Lemoore and Tomelloso separately and (b) Lemoore and Tomelloso when separated into two datasets. Calibration phase of the Whit04 dataset for (c) all the sample, (d) the thirty soil samples from Tomolleso and the thirty soil samples from Lemoore and (e) the thirty soil samples from Tomelloso soils divided into two datasets.

To validate this method of classification, a cross validation was performed on the Whit04 dataset

by dividing the Lemoore soils into two subsets randomly chosen and equally sized, one for the
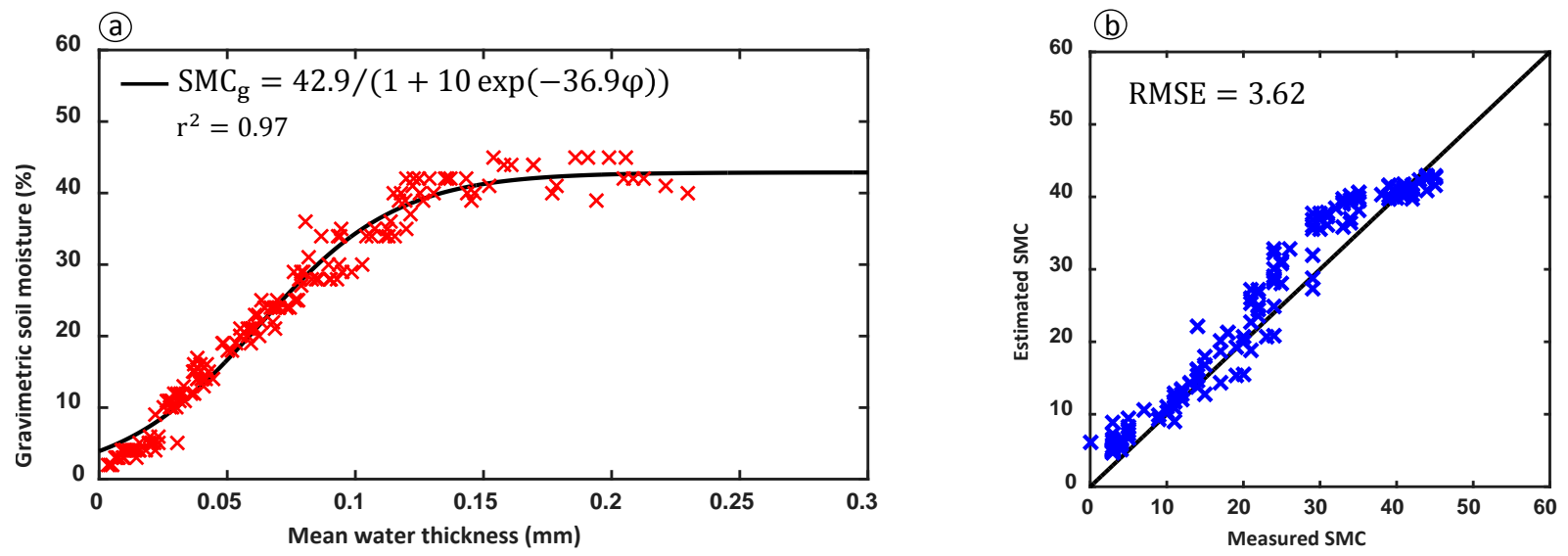

Fig. 16. (a) Calibration phase on eight soils from Lemoore and (b) prediction step on seven other soils of this same area.

We extended the study to the Lob02, Whit04, Les08 and Bab16 datasets all together. At this stage Liu02, Mar12 and Phil14 have been discarded due to different measurement protocols. Using the classification of Lacerda et al. (2016), three groups have been defined (Fig. 17a): group I contains a mixture of clayey and sandy soils, group II contains sandy soils and group III contains very sandy 
471 accuracy. Moreover, the coefficient $K$ of the group III, which is made of sandy soils, is lower than that
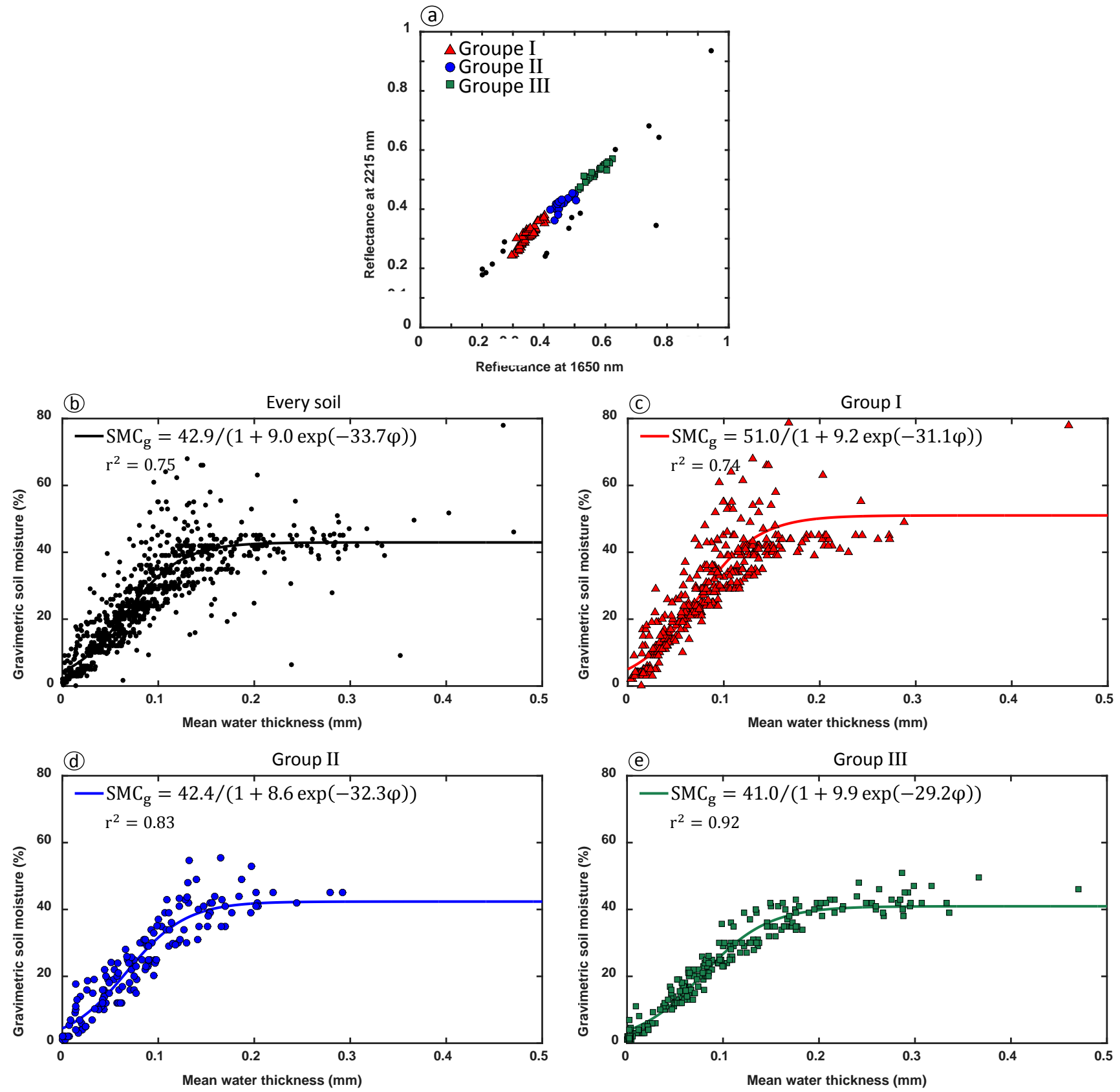

Fig. 17. (a) Lacerda classification method applied to ninety-seven soils of Lob02, Whit04, Les08 and Bab16, black dots are the unclassified samples. Calibration step on (b) all the soils of the datasets Lob02, Whit04, Les08 and Bab16, (c) fiftythree soils of group I, (d) twenty-two soils of group II and (e) the twenty-five soils of group III.

This method of classification seems to be adequate to build calibration equations that are strong enough to use MARMITforSMC globally. The next step is to use it outdoor, i.e., to see to what extent the atmospheric absorption bands affect the SMC retrieval. 
479

480

481

482

\subsection{MARMIT and MARMITforSMC without atmospheric absorption bands}

In real conditions, part of the solar spectrum is not available because of atmospheric absorption bands. We removed the two main bands located between $1300 \mathrm{~nm}$ and $1500 \mathrm{~nm}$ and between $1800 \mathrm{~nm}$ and $2100 \mathrm{~nm}$, and inverted MARMIT again. Fig. 18 shows a good fit of the data, which is not surprising since Bach and Mauser (1994) came to the same conclusion with their model.

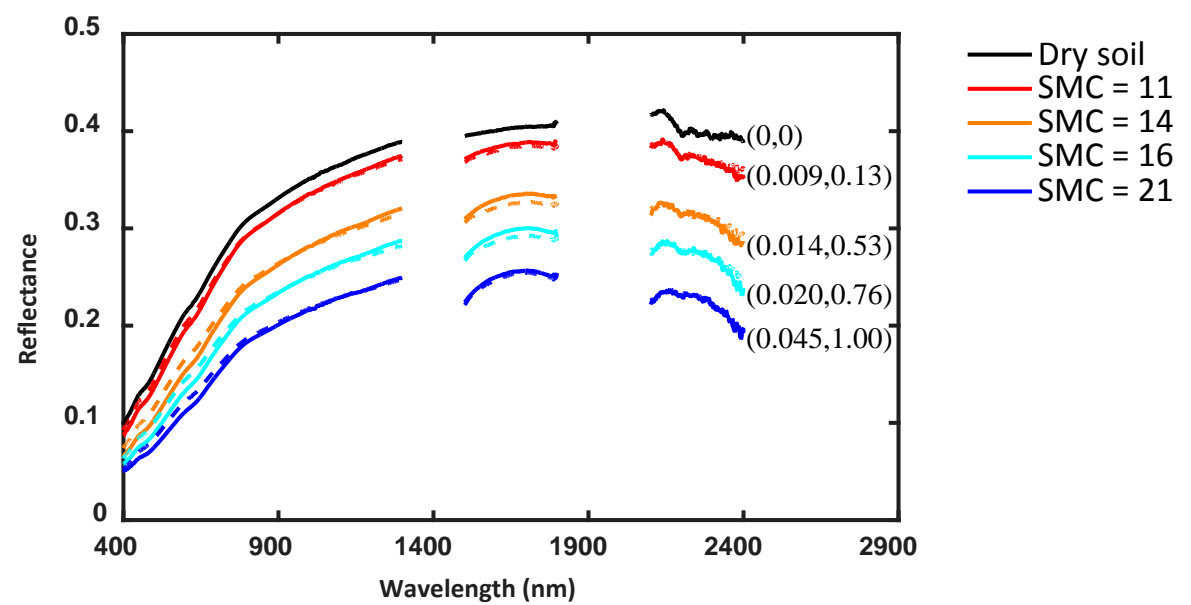

Fig. 18. Measured (solid line) and modeled (dashed line) reflectance spectra, excluding data in the main atmospheric absorption bands of one soil at various levels of soil water content: soil from Ithaca in Phil14. The retrieved parameters of the model are provided to the right of the curves $(L, \varepsilon) . L$ is in $\mathrm{mm}$.

The RMSE obtained without the atmospheric absorption bands is even better (Fig. 19). The mean water thickness estimated by inversion of MARMIT is very similar.

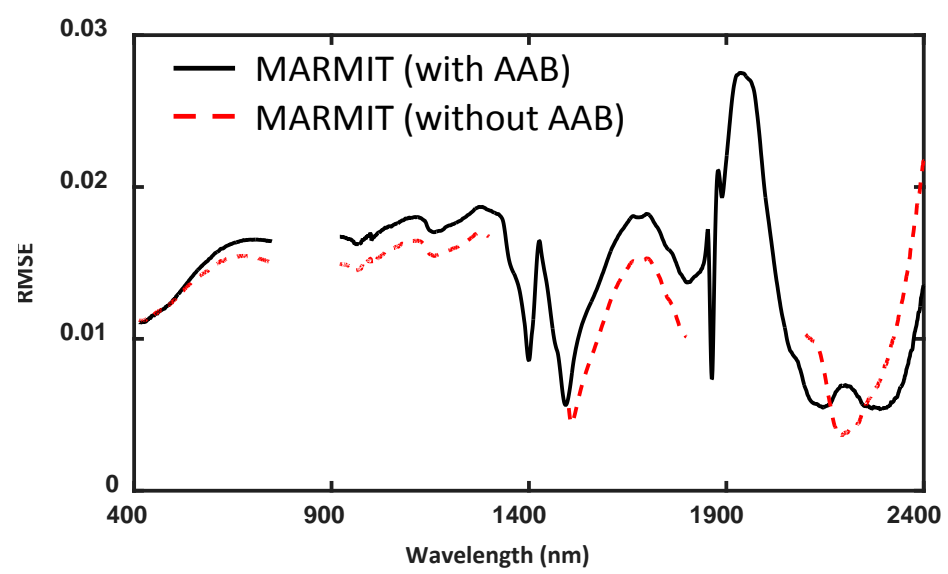

Fig. 19. RMSE obtained with MARMIT with and without reflectance data in the main atmospheric absorption bands (AAB) for all the soils of all the database. 
In this section, the MARMITforSMC method described in Section 3.2 is compared to other

492

493

494

495

496

497

498

499

500

501

502

503

504

505

methods published in the literature for soil moisture content estimation. As they require calibration, we performed the calibration step and the assessment step on the same dataset as in Sadeghi et al. (2015).

\subsection{Semi-empirical methods}

Five spectral indices and two semi-empirical approaches, that are the Soil Moisture Gaussian Model (SMGM) (Whiting et al., 2004) and the relative absorption depth (RAD) through Continuum Removal method, were investigated. A spectral index is a combination of reflectances at two or more wavelengths: generally one chooses wavelengths where photons are not or little absorbed and wavelengths where, contrariwise, they are strongly absorbed. Most of the spectral indices listed in Table 3 are typically used to determine soil water content. They perform quite well and are easy to use.

\begin{tabular}{lcc}
\hline \multicolumn{1}{c}{ Index } & Formula & Source \\
\hline Normalized Soil Moisture Index (NSMI) & $\frac{R_{1800}-R_{2119}}{R_{1800}+R_{2119}}$ & Haubrock et al. (2008) \\
\hline $\begin{array}{l}\text { Normalized Index of NSWIR domain for } \\
\begin{array}{l}\text { SMC estimation from Linear regression } \\
\text { (NINSOL) }\end{array}\end{array}$ & $\frac{R_{2076}-R_{2230}}{R_{2076}+R_{2230}}$ & Fabre et al. (2015) \\
\hline $\begin{array}{l}\text { Normalized Index of NSWIR domain for } \\
\begin{array}{l}\text { SMC estimation from Non-linear regression } \\
\text { (NINSON) }\end{array}\end{array}$ & $\frac{R_{2122}-R_{2230}}{R_{2122}+R_{2230}}$ & Oltra-Carrió et al. (2015) \\
\hline $\begin{array}{l}\text { Normalized Difference Water Index } \\
\text { (NDWI) }\end{array}$ & $\frac{R_{860}-R_{1240}}{R_{860}+R_{1240}}$ & Gao (1996) \\
\hline Water Index SOIL (WISOIL) & $\frac{R_{1450}}{R_{1300}}$ & Whalley et al. (1991) \\
\hline
\end{tabular}

Table 3. Some spectral indices found in the literature. Wavelengths are expressed in nm.

The SMGM and RAD methods are based on the continuum of the spectrum. Indeed, absorption features in the reflectance spectrum can be isolated by a mathematical function called apparent continuum (Clark and Roush, 1984). In the SMGM, Whiting et al. (2004) fit an inverted Gaussian function to the continuum and they calibrate the area below the curve to SMC. Yin et al. (2013) calculate the relative absorption depth as $R=1-R_{b} / R_{c}$ (where $R_{b}$ is the normalized reflectance at 
$5111940 \mathrm{~nm}$ and $R_{c}$ the one at $1800 \mathrm{~nm}$ ) and they regress it against SMC. In order to have a reliable

512 calibration, the results provided by these methods are compared to MARMITforSMC for datasets

513 containing more than five soil samples (Fig. 20). The worst results are obtained with the Liu02 and

514 Bab16 datasets, due to the nature of the soils described above. Let's now analyze the methods 515 individually:

516 1) The indices NSMI, NINSOL and NINSON which are known to perform well (Fabre et al., 2015)

517 provide the best results contrary to NDWI index which is mainly used for the detection of water in 518 vegetation (Gao, 1996; Khanna et al., 2007). NSMI, which performs best with Liu02, a dataset 519 containing oversaturated samples, seems to be the most appropriate index for saturated soils and 520 WISOIL also gives good results. But none of these both indices can be used in remote sensing 521 because one of the wavelength used is located in a broad atmospheric absorption band.

2) SMGM works well on Whit04 and Mar12 because the efficiency of this method depends on the spectral shape of soil reflectance, which is very similar in these two datasets. The main disadvantage of this method is that it is limited to soil below saturation with water content less than 0.32 g/g (Whiting et al., 2004).

3) The RAD method always leads to acceptable errors but never performs the best. It means that it is robust but less efficient than NSMI, WISOIL and MARMITforSMC. Moreover, it has only been tested on samples in laboratory conditions and it uses a wavelength located in one of the main atmospheric absorption band (1940 nm).

4) MARMITforSMC performs very well and is the most robust method except for the Liu02 dataset for reasons already discussed. 

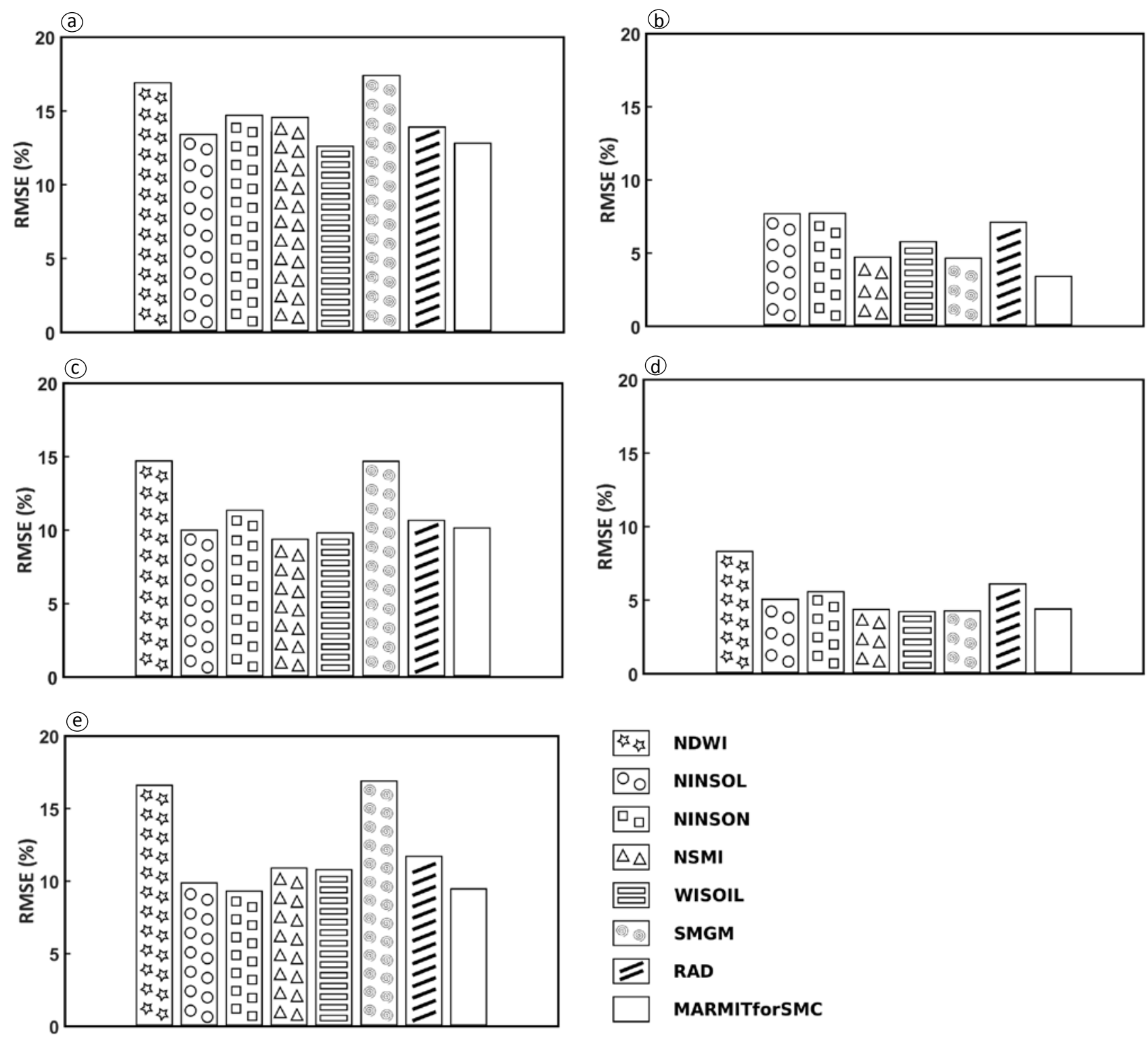

Fig. 20. Comparison of RMSE obtained with the spectral indices of Table 3, the SMGM and RAD methods, and MARMITforSMC using the datasets: (a) Liu02, (b) Whit04, (c) Les08, (d) Mar12 and (e) Bab16. NDWI cannot be applied to Whit04 because the reflectance at $860 \mathrm{~nm}$ is not available.

\subsection{Physical models}

\subsubsection{The Sadeghi model}

Sadeghi et al. (2015) developed a model based on the Kubelka-Munk theory. They provide two

538 reflectance of the wet soil $(r)$, the dry soil $\left(r_{d}\right)$, the saturated soil $\left(r_{s}\right)$ and to the saturated SMC 539 (SMCs):

$$
S M C=\frac{r-r_{d}}{r_{s}-r_{d}} S M C_{s}
$$



and MODIS bands) by the relation $r=(1-R)^{2} / 2 R$. Sadeghi et al. (2015) tested the efficiency of the simplified model for SMC retrieval on two datasets: Lob02 and Whit04. For Lob02 they tested it soil-

545 by-soil and for Whit04 they separated the Lemoore soils from Tomelleso soils. They first performed a calibration step: SMCs are measured and they obtain $r_{d}$ and $r_{s}$ thanks to a least square optimization. Then they used this calibration equation to assess SMC. Table 4 provides the RMSE of SMC retrieved using the Sadeghi model and using MARMITforSMC.

\begin{tabular}{cccc}
\hline \multirow{2}{*}{ Dataset } & \multirow{2}{*}{ Soil } & \multicolumn{2}{c}{ RMSE (\%) } \\
\cline { 3 - 4 } & & Sadeghi & MARMITforSMC \\
\hline \multirow{3}{*}{ Lob02 } & Andisol & 3.6 & $\mathbf{2 . 4}$ \\
& Entisol & $\mathbf{1 . 2}$ & 2.4 \\
& Aridosol & $\mathbf{0 . 5}$ & 1.1 \\
& Mollisol & 3.0 & $\mathbf{2 . 8}$ \\
\hline \multirow{2}{*}{ Whit04 } & Lemoore & 6.7 & $\mathbf{2 . 7}$ \\
& Tomelloso & 7.7 & $\mathbf{3 . 3}$ \\
\hline
\end{tabular}

Table 4. Comparison of SMC assessment with MARMIT compared to the one with the Sadeghi model. In bold the best results.

MARMITforSMC performs much better than the Sadeghi model for the Whit04 dataset while the results are similar for the Lob02 dataset, depending on the soil. The RMSE are divided by more than two because, in the calibration phase of the Sadeghi model, only one transformed reflectance spectrum of dry and wet soil can be obtained by inversion even if different soils are studied. In MARMIT the reflectance spectrum of the dry soil is used for each soil.

\subsubsection{The Bach model}

As mentioned in Section 2.1, MARMIT derives from the Bach model (Eq. (5)). Bach (1995) also performs a calibration of her model but she relates SMC with $L$ using a linear regression. We decided to apply the same calibration phase as in MARMITforSMC and to link $\varphi$ and SMC with a sigmoid function. The entire dataset Whit04 and the three classes of Les08 have been compared. MARMIT leads to a better RMSE (Table 5), even if the Bach model better fits the measured reflectance spectra (Fig. 6) due to an adapted water absorption coefficient. 


\begin{tabular}{cccc}
\hline \multirow{2}{*}{ Dataset } & \multicolumn{2}{c}{ RMSE (\%) } \\
\cline { 3 - 4 } Whit04 & & Bach & MARMITforSMC \\
\cline { 3 - 4 } & & 4.8 & $\mathbf{3 . 4}$ \\
\hline \multirow{3}{*}{ Les08 } & Whole dataset & 7.1 & $\mathbf{6 . 7}$ \\
& Class I & 7.0 & $\mathbf{6 . 7}$ \\
& Class II & 4.8 & $\mathbf{4 . 3}$ \\
& Class III & 2.9 & $\mathbf{2 . 1}$ \\
\hline
\end{tabular}

Table 5. Comparison between MARMIT and the Bach model. In bold the best RMSE.

\section{Conclusion}

A simple physically-based model called MARMIT was developed and used for SMC retrieval on seven independent datasets gathering 217 soil samples collected in China, France, Spain, Tunisia and the U.S.A. The model estimates SMC in three key steps which constitutes the MARMITforSMC method: (1) inversion, (2) calibration, and (3) (cross-)validation. Step (1) works better for low SMC values. Sometimes it is difficult to fit the reflectance in the visible probably because some phenomena are not taken into account: for instance, it is likely that the soil particles and the water film mix as suggested by Philpot (2010). This leads to an overestimation of the reflectance outside the water absorption bands and an underestimation in the water absorption bands. Despites this issue, step (2) explains very well the evolution of SMC with $\varphi$ when soils are considered individually $\left(r^{2} \geq 0.95\right)$. The variation of soil moisture content as a function of the mean water thickness is well described by a sigmoid function, some parameters of which are related to soil chemical and physical properties. The parameter $K$ (maximum value of the function) seems to be linked with the SMC at saturation; $a$ (the place of the curve on the $\mathrm{x}$-axis) and $\psi$ (the slope between the two horizontal asymptotes) may be related to mineralogy. Unfortunately, we lack metadata to support this hypothesis. As the goal of the method is to infer the SMC using remote sensing data regardless of the soil type, a soil-by-soil calibration is not desirable. We successfully grouped the soils together into general classes to infer accurate global calibration equations. The most operational way of classification proposed by Lacerda et al. (2016) has been successfully tested on the thirty Lemoore soils of the Whit04 dataset (RMSE $=$ 3.62\%) and more generally on the soils of the datasets Lob02, Whit04, Les08 and Bab16 together. 
Indeed, we found three groups with coefficients of determination of the relationship between SMC

587

588

589

590

591

592

593

594

595

596

597

598

599

600

601

602

603

604

605

606

607

608

609

610

611

612

and $\varphi$ greater than 0.74. Finally, MARMITforSMC has been compared to existing methods for SMC retrieval: the method performs as well or better than other methods, especially the one relying on the Bach model. Moreover, we proved that SMC could be retrieved with the same accuracy as TDR measurements thanks to an appropriate classification (RMSE 3\%). The new method clearly led to an improvement in the SMC retrieval. The main advantage of this model is that it is easy to understand and fast to compute, and there is room for progress. The drawback of MARMITforSMC is that it requires a calibration step, which is soil dependent, and the reflectance spectrum of the dry soil. The first issue can be overcome with a soil classification based on spectral signatures and the second one by multi-data imagery. We also showed that MARMIT was not suitable for oversaturated soils in water. The model may be improved by taking into account specular reflectance. The measurement protocol, e.g., water distribution within the sample, probably influences the results of MARMITforSMC given that the SMC in the sample is variable vertically and horizontally. Future research in SMC retrieval using MARMIT with soils of known textural and mineralogical properties will help extend and improve the model for porosity or grain size.

\section{Acknowledgements}

The PhD thesis of Aurélien Bablet is funded by ONERA and IPGP. This work is also supported by the PNTS (Programme national de télédétection spatiale) in the frame of the SOILSPECT-2 project. The authors would like to thank Sébastien Marcq from CNES (Centre national d'études spatiales), Jean-Marc Gilliot and Emmanuelle Vaudour from INRA (Institut national de la recherche agronomique), Cécile Gomez from LISAH (Laboratoire d'étude des interactions sol-agrosystèmehydrosystème), Véronique Carrère from LPGN (Laboratoire de planétologie et géodynamique de Nantes) and Rodolphe Marion from CEA (Commissariat à l'énergie atomique et aux énergies alternatives) who provided us with the soil samples that make the Bab16 dataset. We also thank William D. Philpot from Cornell University who proofread the article and shared his data. 
614

615

616

617

618

619

620

621

622

623

624

625

626

627

628

629

630

631

632

633

634

635

636

637

638

639

640

641

642

643

644

645

646

647

648

649

650

651

652

653

654

655

656

657

658

659

660

661

662

663

664

Ångström A. (1925), The albedo of various surfaces of ground, Geografiska Annaler, 7:323-342.

Bach H. and Mauser W. (1994), Modeling and model verification of the spectral reflectance of soils under varying moisture conditions, 14th International Geoscience and Remote Sensing Symposium (IGARSS'94), Pasadena (CA), 8-12 August 1994, IEEE, Vol. 4, pp. 2354-2356.

Bach H. (1995), Die Bestimmung hydrologischer und landwirtschaftlicher Oberflächenparameter aus hyperspektralen Fernerkundungsdaten, Münchener Geographische Abhandlungen, München (Germany), pp 90-103.

Baumgardner M.F., Silva L.F., Biehl L.L. and Stoner E.R. (1985), Reflectance properties of soils, Advances in Agronomy, 38:1-44.

Bedidi A., Cervelle B., Madeira J. and Pouget M. (1992), Moisture effects on visible spectral characteristics of lateritic soils, Soil Science, 153(2):129-141.

Ben-Dor E. (2011), Characterization of soil properties using reflectance spectroscopy, in Hyperspectral Remote Sensing of Vegetation (Thenkabail A., Lyon P.S. \& Huete J.G., eds), CRC Press, pp. 513-558.

Bishop J.L., Pieters C.M. and Edwards J.O. (1994), Infrared spectroscopic analyses on the nature of water in montmorillonite, Clays and Clay Minerals, 42(6):702-716.

Bogrekci I. and Lee W.S. (2006), Effects of soil moisture content on absorbance spectra of sandy soils in sensing phosphorus concentrations using UV-VIS-NIR spectroscopy, Transactions of the ASABE, 49(4):1175-1180.

Bowers S.A. and Hanks J. (1965), Reflection of radiant energy from soils, Soil Science, 100(2):130138.

Bryant R., Thomas D., Moran S., Holifield C., Goodrich D., Keefer T., Paige G., Williams D. and Skirvin S. (2003), Evaluation of hyperspectral, infrared temperature and radar measurements for monitoring surface soil moisture, Proceedings of the First Interagency Conference on Research in the Watersheds, 27-30 October 2003, Benson (AZ), pp. 528-533.

Clark R. and Roush T. (1984), Reflectance spectroscopy: quantitative analysis techniques for remote sensing applications, Journal of Geophysical Research, 89:6329-6340.

Das N.N., Mohanty B.P. and Njoku E.G. (2008), Characterization of backscatter by surface features in L-band active microwave remote sensing of soil moisture, In Geoscience and Remote Sensing Symposium (IGARSS'08), IEEE, vol. 2 pp II-817.

Demattê J.A.M (2002), Characterization and discrimination of soils by their reflected electromagnetic energy, Brazilian Journal of Agriculture Research, 37:1445-1458.

Eisenberg D. and Kauzman W. (2005), The Structure and Properties of Water, Oxford University Press, 310 pp.

Fabre S., Briottet X. and Lesaignoux A. (2015), Estimation of soil moisture content from the spectral reflectance of bare soils in the 0.4-2.5 $\mu \mathrm{m}$ domain, Sensors, 15(2):3262-3281.

Gao B. (1996), NDWI-A normalized difference water index for remote sensing of vegetation liquid water from space, Remote Sensing of Environment, 58(3):257-266.

Gao Z., Xu X., Wang J., Yang H., Huang W. and Feng H. (2013), A method of estimating soil moisture based on the linear decomposition of mixture pixels, Mathematical and Computer Modelling, 48(3-4):606-613.

Gardner C.M.K., Robinson D., Blyth K. and Cooper D. (2000), Soil water content, in Soil and Environmental Analysis: Physical Methods, Revised, and Expanded, CRC Press, pp. 1-64.

Glenn E., Mckeon C., Gerhart V., Nagler P., Jordan F. and Artiola J. (2009), Deficit irrigation of a landscape halophyte for reuse saline waste water in a desert city, Landscape and Urban Planning, 89:57-64.

Haubrock S.N., Chabrillat S., Lemmitz C. and Kaufmann H. (2008), Surface soil moisture quantification models from reflectance data under field conditions, International Journal of Remote Sensing, 29(1):3-29.

Idso S.B., Jackson R.D., Reginato R.J., Kimball B.A. and Nakayama F.S. (1975), The dependence of bare soil albedo on soil water content, Journal of Applied Meteorology, 14(1):109-113. 
Ishida T., Ando H. and Fukuhara M. (1991), Estimation of complex refractive index of soil particles and its dependence on soil chemical properties, Remote Sensing of Environment, 38:173-182.

Kaleita A.L., Tian L.F. and Hirschi M.C. (2005), Relationship between soil moisture content and soil surface reflectance, Transactions of the ASAE, 48(5):1979-1986.

Khanna S., Palacios-Orueta A., Whiting M.L., Ustin S.L., Riaño D. and Litago J. (2007), Development of angle indexes for soil moisture estimation, dry matter detection and land-cover discrimination, Remote Sensing of Environment, 109:154-165.

Kimmel B.W. and Baranoski G.V.G. (2007), A novel approach for simulating light interaction with particulate materials: application to the modeling of sand spectral properties, Optics Express, 15(15):9755-9777.

Lacerda M.P.C., Demattê J.A.M., Sato M.V., Fongaro C.T., Gallo B.C. and Souza A.B. (2016), Tropical texture determination by proximal sensing using a regional spectral library and its relationship with soil classification, Remote Sensing, 8(9):701.

Lekner J. and Dorf M.C. (1988), Why some things are darker when wet, Applied Optics, 27(7):12781280.

Lesaignoux A. (2010), Estimation de l'humidité de surface des sols nus à partir de l'imagerie hyperspectrale à haute résolution spatiale sur le domaine optique 0,4-14 $\mu \mathrm{m}$, $\mathrm{PhD}$ Thesis, Université de Toulouse, 203 pp.

Lesaignoux A., Fabre S. and Briottet X. (2013), Influence of soil moisture content on spectral reflectance of bare soils in the 0.4-14 $\mu \mathrm{m}$ domain, International Journal of Remote Sensing, 34(7):2268-2285.

Levitt D.G., Simpson J.R. and Huete A.R. (1990), Estimates of surface soil water content using linear combinations of spectral wavebands, Theoretical and Applied Climatology, 42(4):245-252.

Liu W., Baret F., Gu X.F., Tong Q., Zheng L. and Zhang B. (2002), Relating soil moisture to reflectance, Remote Sensing of Environment, 81(2-3):238-246.

Liu W., Baret F., Gu X.F., Zhang B., Tong Q. and Zheng L. (2003), Evaluation of methods for soil surface moisture estimation from reflectance data, International Journal of Remote Sensing, 24(10):2069-2083.

Lobell B.B. and Asner G.P. (2002), Moisture effects on soil reflectance, Soil Science Society of America Journal, 66(3):722-727.

Marcq S. (2012), Développement d'un outil end-to-end permettant de modéliser la signature spectrale de la végétation au sommet de l'atmosphère, Rapport d'activité IPGP-CEA, 50 pp.

Marion R. and Carrère V. (2018), Mineral Mapping Using the Automatized Gaussian Model (AGM)-Application to Two Industrial French Sites at Gardanne and Thann, Remote Sensing, 10(1):146.

Milliken R.E. and Mustard J.F. (2005), Quantifying absolute water content of minerals using nearinfrared reflectance spectroscopy, Journal of Geophysical Research - Planets, 110(E12).

Milliken R.E. and Mustard J.F. (2007a), Estimating the water content of hydrated minerals using reflectance spectroscopy I. Effects of darkening agents and low-albedo materials, Icarus, 189:550573.

Milliken R.E. and Mustard J.F. (2007b), Estimating the water content of hydrated minerals using reflectance spectroscopy II. Effects of particle size, Icarus, 189:574-588.

Minasny B., McBratney A.B., Bellon-Maurel V., Roger J.M., Gobrecht A., Ferrand L. and Joalland S. (2011), Removing the effect of soil moisture from NIR diffuse reflectance spectra for the prediction of soil organic carbon, Geoderma, 167-168:118-124.

Mira M., Valor E., Boluda R., Caselles V. and Coll C. (2007), Influence of soil water content on the thermal infrared emissivity of bare soils: implication for land surface temperature determination, Journal of Geophysical Research, 112: F04003.

Mladenova I.E., Jackson T.J., Njoku E., Bindlish R., Chan S., Cosh M.H., Holmes T.R.H., De Jeu R.A.M., Jones L., Kimball J. and Paloscia S. (2014), Remote monitoring of soil moisture using passive microwave-based techniques-Theoretical basis and overview of selected algorithms for AMSR-E. Remote Sensing of Environment, 144:197-213.

Mouazen A.M., Karoui R., De Baerdemaeker J. and Ramon H. (2006), Characterization of soil water 
content using measured visible and near infrared spectra, Soil Science Society of America Journal, 70(4):1295- 1302.

Muller E. and Décamps H. (2001), Modeling soil moisture-reflectance, Remote Sensing of Environment, 76(2):173-180.

Narayanan R., Green S. and Alexander D. (1993), Mid-infrared laser reflectance of moist soils, Applied Optics, 32(30):6043-6052.

Neema D.L., Shah, A. and Patel A.N. (1987), A statistical optical model for light reflection and penetration through sand, International Journal of Remote Sensing, 8(8):1209-1217.

Nelder J. and Mead R. (1965), A simplex method for function minimization, The Computer Journal, 7(4):308-313.

Njoku E.G. and Entekhabi D. (1996). Passive microwave remote sensing of soil moisture, Journal of hydrology, 184(1-2):101-129.

Ochsner T., Cosh M., Cuenca R., Dorigo W., Draper C., Hagimoto Y., Kerr Y., Njoku E., Small E., Zreda M. and Larson K. (2013), State of the art in large-scale soil moisture monitoring, Soil Science Society of America Journal, 77(6):1888-1919.

Okin G. and Painter T. (2004), Effect of grain size on remotely sensed spectral reflectance of sandy desert surfaces, Remote Sensing of Environment, 89:272-280.

Palmer K. and Williams D. (1974), Optical properties of water in the near infrared, Journal of the Optical Society of America, 64:1107-1110.

Patel N.R., Anapashsha R., Kumar S., Saha S. and Dadhwal V. (2009), Assessing potential of MODIS derived temperature/vegetation condition index (TVDI) to infer soil moisture status, International Journal of Remote Sensing, 30(1):23-39.

Peng J., Shen H., He S.W. and Wu J.S. (2013), Soil moisture retrieving using hyperspectral data with the application of wavelet analysis, Environmental Earth Sciences, 69(1):279-288.

Petropoulos G.P., Griffiths H.M., Dorigo W., Xaver A. and Gruber A. (2013), Surface soil moisture estimation: significance, controls, and conventional measurement techniques, in Remote Sensing of Energy Fluxes and Soil Moisture Content (Petropoulos G.P., ed), CRC Press, pp. 29-48.

Philpot W. (2010), Spectral reflectance of wetted soils, in Proceedings of Art, Science and Applications of Reflectance Spectroscopy (ASARS) Symposium, Boulder, CO, USA, Vol. II, 11 pp.

Planet W.G. (1970), Some comments on reflectance measurements of wet soils, Remote Sensing of Environment, 1(2):127-129.

Pommerol A., Schmitt B., Beck P. and Brissaud O. (2009), Water sorption on Martian regolith analogs: thermodynamics and near-infrared reflectance spectroscopy, Icarus, 204(1):114-136.

Pommerol A., Thomas N., Jost B., Beck P., Okubo C. and McEwen A.S. (2013), Photometric properties of Mars soils analogs, Journal of Geophysical Research - Planets, 118(10):2045-2072.

Rienzi E.A., Mijatovic B., Mueller T.G., Matocha C.J., Sikora F.J. and Castrignanò A.M. (2014), Prediction of soil organic carbon under varying moisture levels using reflectance spectroscopy, Soil Science Society of America Journal, 78(3):958-967.

Robinson D., Campbell C., Hopmans J., Hornbuckle B., Jones S., Knight R., Ogden F., Selker J. and Wendroth O. (2008), Vadose Zone Journal, 7(1):358-389.

Rodionov A., Pätzold S., Welp G., Cañada Pallares R., Damerow L. and Amelun W. (2014), Sensing of soil organic carbon using visible and near-infrared spectroscopy at variable moisture and surface roughness, Soil Science Society of America Journal, 78(3):949-957.

Sadeghi M., Jones S.B. and Philpot W.D. (2015), A linear physically-based model for remote sensing of soil moisture using short wave infrared bands, Remote Sensing of Environment, 164:66-76.

Sadeghi M., Babaeian E., Tuller M., and Jones S.B. (2017), The optical trapezoid model: a novel approach to remote sensing of soil moisture applied to Sentinel-2 and Landsat-8 observations, Remote Sensing of Environment, 198:52-68.

Skidmore E.L. and Woodruff N.P. (1968), Wind erosion forces in the United States and their use in predicting soil loss, Agriculture Handbook No. 346. U.S. Dept. Agriculture, Agricultural Research Service, pp 42.

Somers B., Gysels V., Verstraeften W.W., Delalieux S. and Coppin P. (2010), Modelling moistureinduced soil reflectance changes in cultivated sandy soils: a case study in citrus orchards, European 
Journal of Soil Science, 61:1091-1105.

Stafford J.V. (1988), Remote, non-contact and in-situ measurement of soil moisture content: a review, Journal of Agricultural Engineering Research, 41:151-172.

Stenberg B., Viscarra Rossel R.A., Mouazen A.M. and Wetterlind J. (2010), Visible and near infrared spectroscopy in soil science, Advances in Agronomy, 107:163-215.

Stern F. (1964), Transmission of isotropic radiation across an interface between two dielectrics, Applied Optics, 3(1):111-113.

Stoner E.R. and Baumgardner M.F. (1981), Characteristic variations in reflectance of surface soils, Soil Science Society of America, 45(6):1161-1165.

Sun B., Sunkavalli K., Ramamoorthi R., Belhumeur P.N. and Nayar S.K. (2007), Time-varying BRDFs, IEEE Transactions on Visualization and Computer Graphics, 13(3):595-609.

Tabatabaeenejad A., Burgin M., Duan X. and Moghaddam M. (2015), P-band radar retrieval of subsurface soil moisture profile as a second-order polynomial: first AirMOSS results, IEEE Transactions on Geoscience and Remote Sensing, 53(2):645-658.

Tian J. and Philpot W.D. (2015), Relationship between surface soil water content, evaporation rate, and water absorption band depths in SWIR reflectance spectra, Remote Sensing of Environment, 169:280-289.

Tian J. and Philpot W.D. (2015), Relating water absorption features to soil moisture characteristics, Department of Civil and Environmental Engineering, Cornell University, Ithaca, NY, USA.

Tuller M., Or D., and Dudley L. M. (1999), Adsorption and capillary condensation in porous media: Liquid retention and interfacial configurations in angular pores, Water Resources Research, 35(7), 1949-1964.

Twomey S.A., Bohren C.F. and Mergenthaler J.L. (1986), Reflectance and albedo differences between wet and dry surfaces, Applied Optics, 25(3):431-437.

Van Bavel C.H.M. and Hillel D.I. (1976), Calculating potential and actual evaporation from a bare soil surface by simulation of concurrent flow of water and heat, Agricultural Meteorology, 17(6):453-476.

Vereecken H., Huisman J.A., Bogena H., Vanderborght J., Vrugt J.A. and Hopmans J.W. (2008), On the value of soil moisture measurements in vadose zone hydrology: A review, Water Resources Research, 44:W00D06.

Verpoorter C., Carrère V. and Combe J.P. (2014), Visible, near-infrared spectrometry for simultaneous assessment of geophysical sediment properties (water, grain size) using the Spectral Derivative - Modified Gaussian Model, Journal of Geophysical Research - Earth Surface, 119(10):2098-2122.

Walker J., Willgoose G. and Kalma J. (2004), In situ measurements of soil moisture: a comparison of techniques, Journal of Hydrology, 293:85-89.

Wang L. and Qu J. (2009), Satellite remote sensing applications for surface soil moisture monitoring: a review, Frontiers of Earth Science in China, 3(2):239-247.

Whiting M.L. (2004), Soil moisture model to improve mineral abundance estimates from hyperspectral data, PhD thesis, Department of Land, Air and Water Resources, University of California, Davis, 136 pp.

Whiting M.L., Li L. and Ustin S.L. (2004), Predicting water content using Gaussian model on soil spectra, Remote Sensing of Environment, 89(4):535-552.

Wozniak B. and Dera J. (2007), Light absorption in sea water, Springer, 453 pp.

Yang Y., Shang S. and Jiang L. (2012), Remote sensing temporal and spatial patterns of evapotranspiration and the responses to water management in a large irrigation district of North China, Agricultural and Forest Meteorology, 164:112-122.

Yin Z., Lei T., Yan Q., Chen Z. and Dong Y. (2013), A near-infrared reflectance sensor for soil surface moisture measurement. Computers and Electronics in Agriculture, 99:101-107.

Zhang Y.F., Wang X.P., Hu R., Pan Y.X. and Zhang H. (2014), Variation of albedo to soil moisture for sand dunes and biological soil crusts in arid desert ecosystems, Environmental Earth Sciences, 71(3):1281-1288. 